\title{
AVTA Federal Fleet PEV Readiness Data Logging and Characterization Study for the National Park Service: Fort Vancouver National Historic Site
}

Stephen Schey Jim Francfort

March 2014

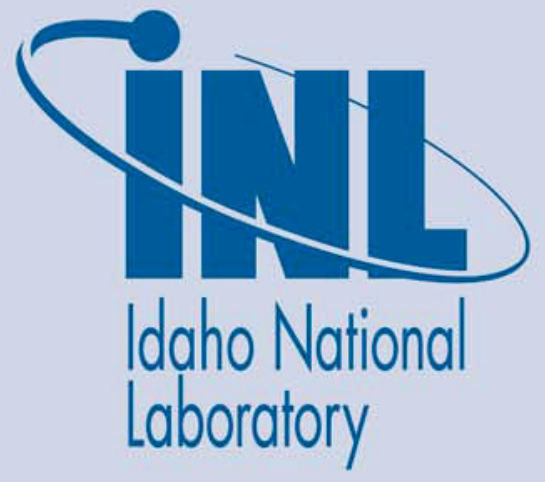

The INL is a U.S. Department of Energy National Laboratory operated by Battelle Energy Alliance 


\section{DISCLAIMER}

This information was prepared as an account of work sponsored by an agency of the U.S. Government. Neither the U.S. Government nor any agency thereof, nor any of their employees, makes any warranty, expressed or implied, or assumes any legal liability or responsibility for the accuracy, completeness, or usefulness, of any information, apparatus, product, or process disclosed, or represents that its use would not infringe privately owned rights. References herein to any specific commercial product, process, or service by trade name, trade mark, manufacturer, or otherwise, does not necessarily constitute or imply its endorsement, recommendation, or favoring by the U.S. Government or any agency thereof. The views and opinions of authors expressed herein do not necessarily state or reflect those of the U.S. Government or any agency thereof. 


\title{
AVTA Federal Fleet PEV Readiness Data Logging and Characterization Study for the National Park Service: Fort Vancouver National Historic Site
}

\author{
Stephen Schey ${ }^{1}$ \\ Jim Francfort ${ }^{2}$ \\ ${ }^{1}$ Stephen Schey, Project Manager, Infrastructure Planning and Analysis; Intertek Testing Services, \\ North America; Phoenix, AZ \\ ${ }^{2}$ Jim Francfort, Vehicle Systems Principal Investigator; Idaho National Laboratory operated by \\ Battelle energy Alliance; Idaho Falls, ID
}

March 2014

Idaho National Laboratory Idaho Falls, Idaho 83415

http://www.inl.gov

Prepared for the

U.S. Department of Energy

Office of Nuclear Energy

Under DOE Idaho Operations Office

Contract DE-AC07-05ID14517 


\section{ABSTRACT}

Battelle Energy Alliance, LLC, managing and operating contractor for the U.S. Department of Energy's Idaho National Laboratory, is the lead laboratory for the U.S. Department of Energy's Advanced Vehicle Testing. Battelle Energy Alliance, LLC contracted with Intertek Testing Services, North America (ITSNA) to collect data on federal fleet operations as part of the Advanced Vehicle Testing Activity's Federal Fleet Vehicle Data Logging and Characterization study. The Advanced Vehicle Testing Activity study seeks to collect data to validate the use of advanced electric drive vehicle transportation.

This report focuses on the Fort Vancouver National Historic Site (FVNHS) fleet to identify daily operational characteristics of select vehicles and report findings on vehicle and mission characterizations to support the successful introduction of electric vehicles (EVs) into the agencies' fleet.

Individual observations of the selected vehicles provided the basis for recommendations related to EV adoption and whether a battery electric vehicle (BEV) or plug-in hybrid electric vehicle (PHEV) (collectively plug-in electric vehicles) could fulfill the mission requirements.

FVNHS identified three vehicles in its fleet for consideration. While the FVNHS vehicles conduct many different missions, only two (i.e., support and pool missions) were selected by agency management to be part of this fleet evaluation. The logged vehicles included a pickup truck and a minivan.

This report will show that BEVs and PHEVs are capable of performing the required missions and providing an alternative vehicle for both mission categories, because each has sufficient range for individual trips and time available each day for charging to accommodate multiple trips per day. These charging events could occur at the vehicle's home base, high-use work areas, or in intermediate areas along routes that the vehicles frequently travel.

Replacement of vehicles in the current fleet would result in significant reductions in emission of greenhouse gases and petroleum use, while also reducing fuel costs.

The Vancouver, Washington area and neighboring Portland, Oregon are leaders in adoption of PEVs in the United States ${ }^{1}$. PEV charging stations, or more appropriately identified as electric vehicle supply equipment, located on the FVNHS facility would be a benefit for both FVNHS fleets and general public use. Fleet drivers and park visitors operating privately owned plug-in electric vehicles benefit by using the charging infrastructure. ITSNA recommends location analysis of the FVNHS site to identify the optimal station placement for electric vehicle supply equipment.

ITSNA recognizes the support of Idaho National Laboratory and ICF International for their efforts to initiate communication with the National Parks Service and FVNHS for participation in this study.

ITSNA is pleased to provide this report and is encouraged by the high interest and support from the National Park Service and FVNHS personnel.

\footnotetext{
${ }^{1}$ http://avt.inel.gov/evproject.shtml\# [accessed April 10, 2014]
} 


\section{CONTENTS}

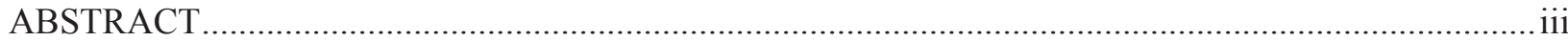

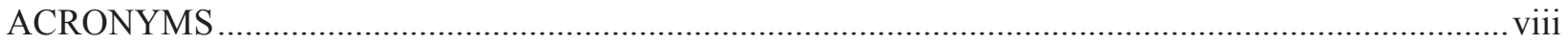

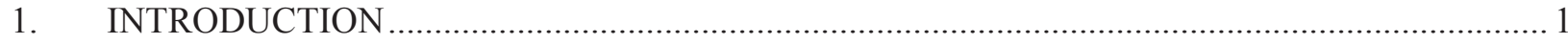

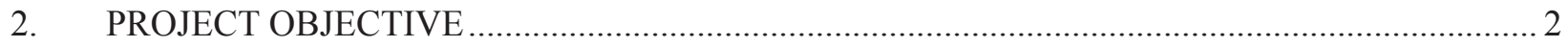

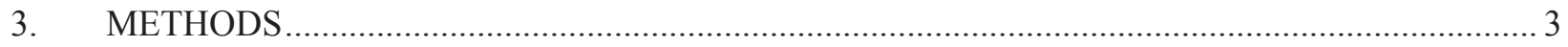

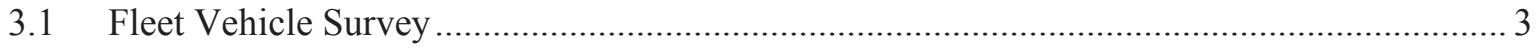

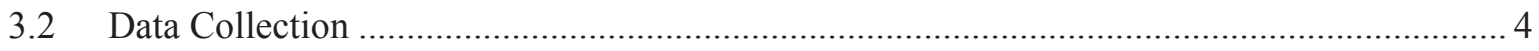

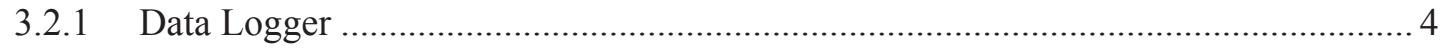

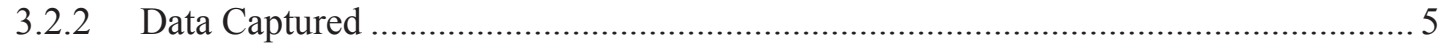

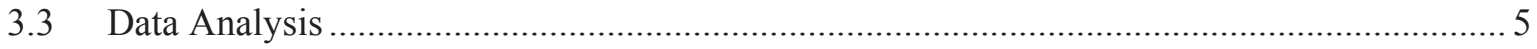

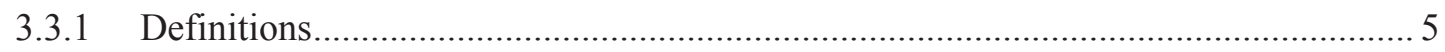

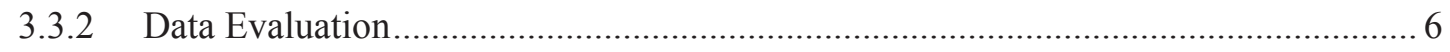

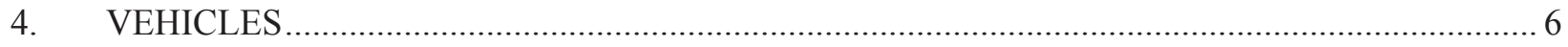

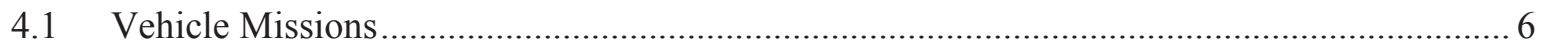

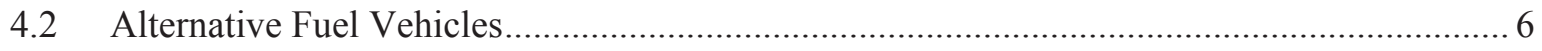

4.3 Battery Electric Vehicle/Plug-in Hybrid Electric Vehicle Benefits/Challenges .................... 7

4.3.1 Battery Electric Vehicle/Electric Vehicle Benefits/Challenges ............................... 8

4.3.2 Plug-in Hybrid Electric Vehicle Benefits/Challenges................................................ 8

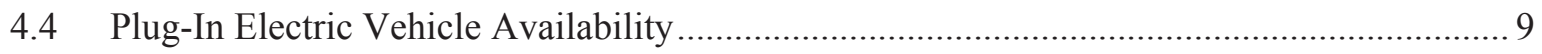

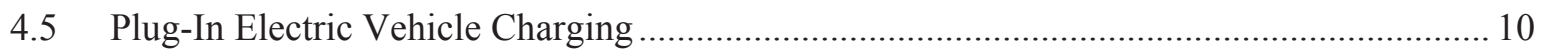

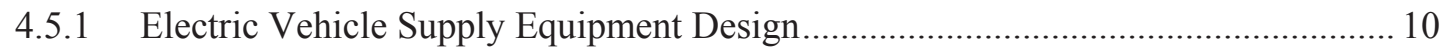

4.5.2 Electric Vehicle Support Equipment Stations .......................................................... 13

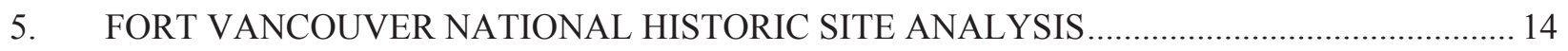

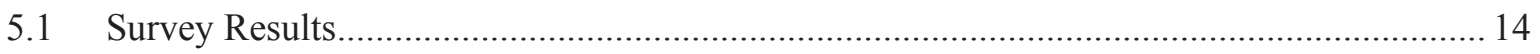

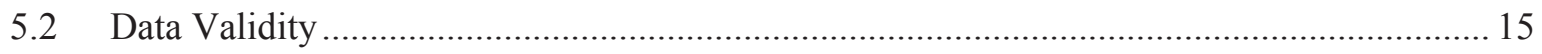

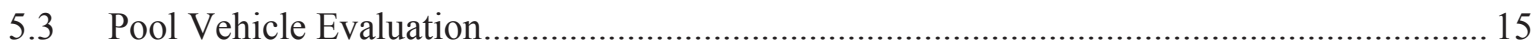

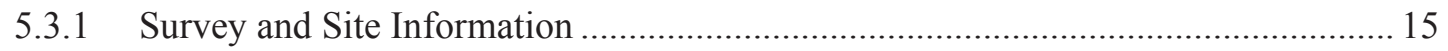

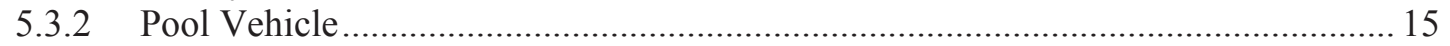




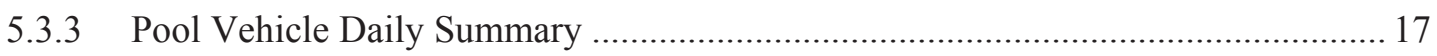

5.3.4 Pool Vehicle Observations/Summary ................................................................. 19

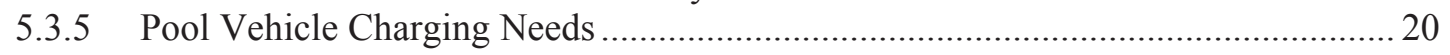

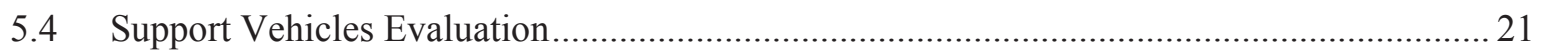

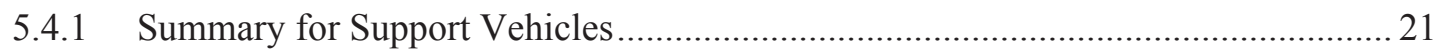

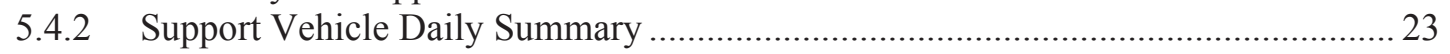

5.4.3 Support Vehicle Observations/Summary …........................................................ 25

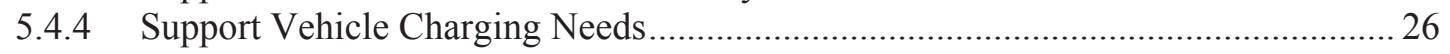

6. GREENHOUSE GAS EMISSIONS AVOIDED AND FUEL COST REDUCTION

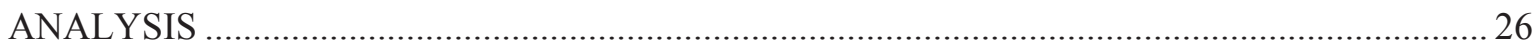

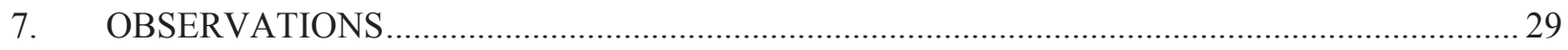

\section{FIGURES}

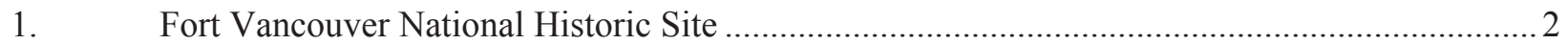

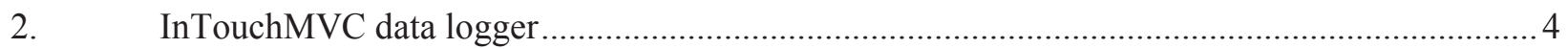

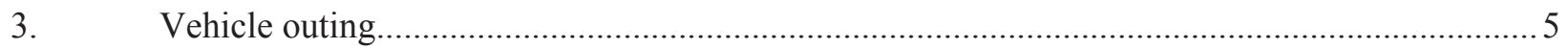

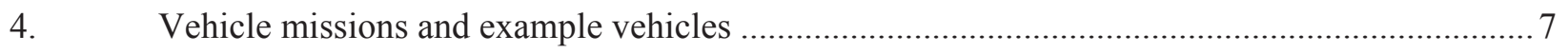

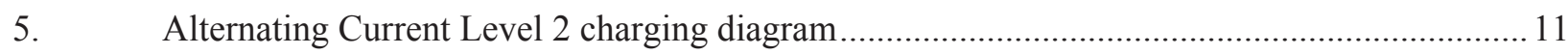

6. Society of Automotive Engineers charging configurations and ratings terminology ................ 12

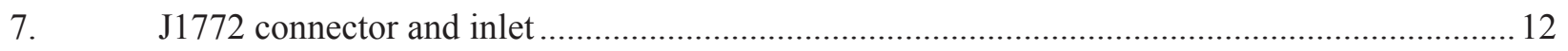

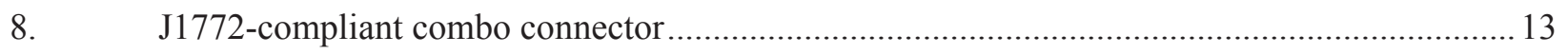

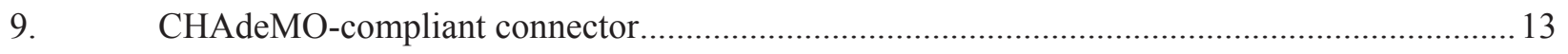

10. Alternating current Level 2 electric vehicle support equipment........................................ 14

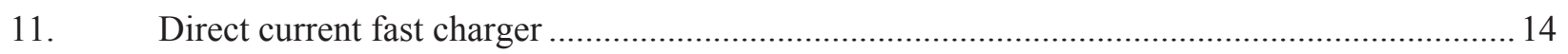

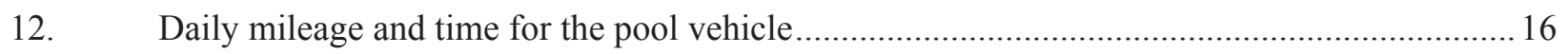

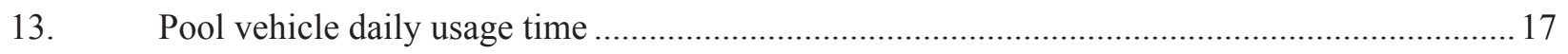

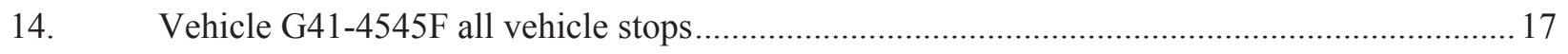

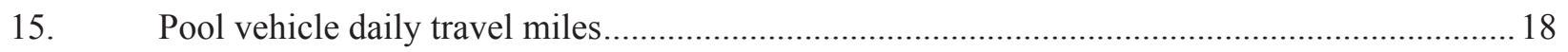

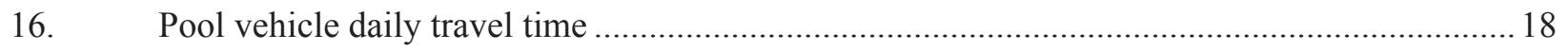




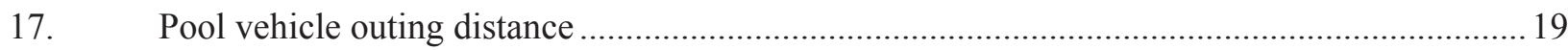

18. Public charging electric vehicle support equipment in the Fort Vancouver National

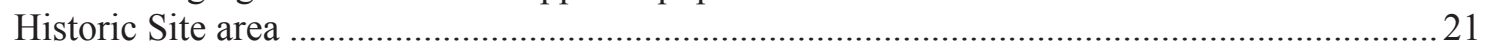

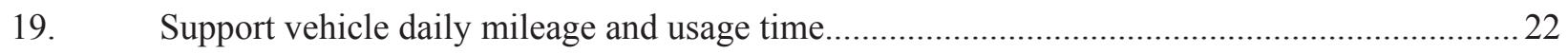

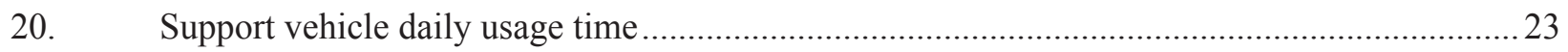

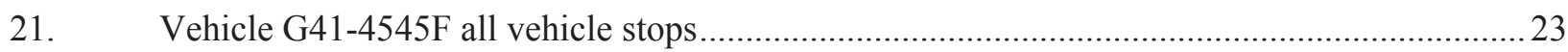

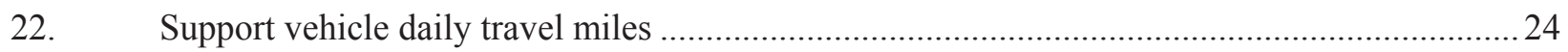

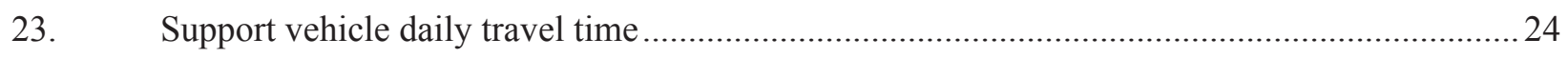

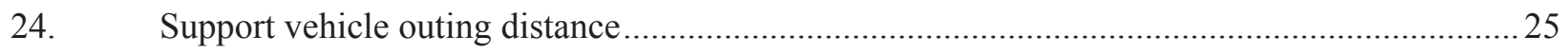

\section{TABLES}

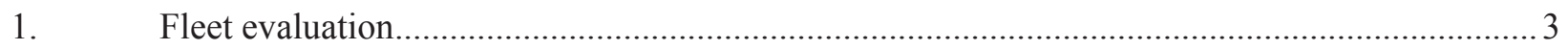

2. General Services Administration vehicle replacement requirements ...................................... 7

3. General Services Administration-certified battery electric vehicles/plug-in electric

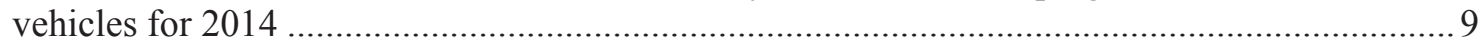

4. Original equipment manufacturer plug-in hybrid electric vehicle cars and availability.............9

5. Original equipment manufacturer battery electric vehicle cars and availability ...................... 10

6. Original equipment manufacturer plug-in hybrid electric vehicle trucks and vans and

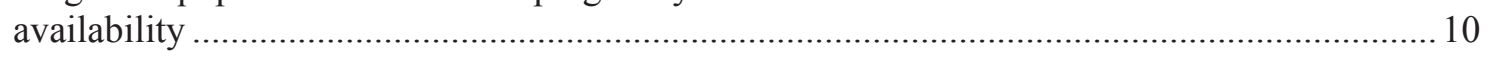

7. Original equipment manufacturer battery electric vehicle trucks and vans and

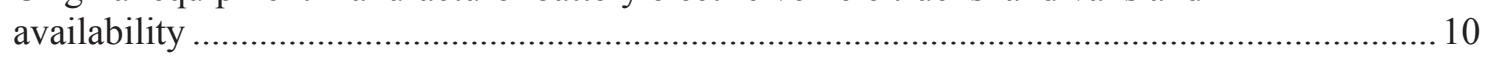

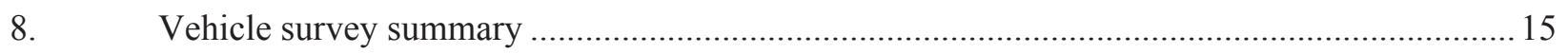

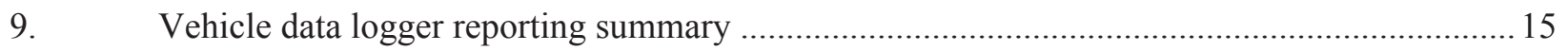

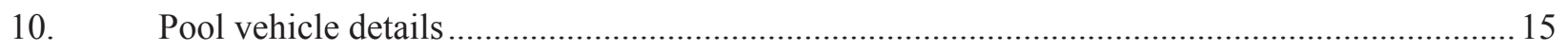

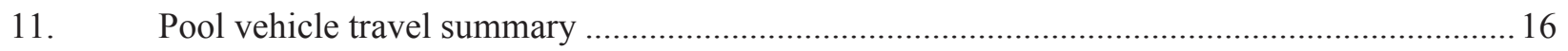

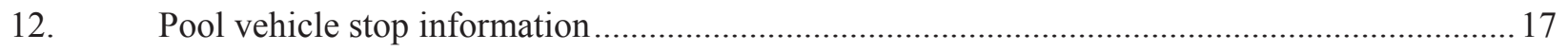

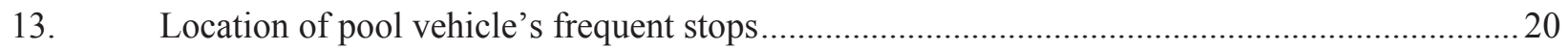

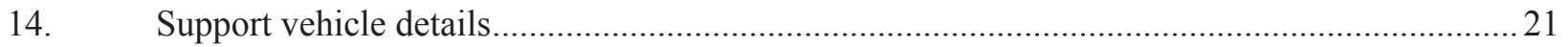




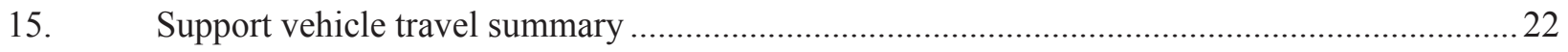

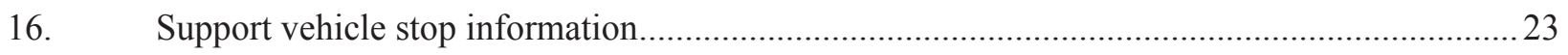

17. Current vehicle replacement plug-in electric vehicles........................................................2 27

18. Environmental Protection Agency internal combustion engine fuel economy ratings..............27

19. Environmental Protection Agency plug-in electric vehicle fuel economy ratings ....................227

20. Greenhouse gas emissions avoidance and fuel cost reduction analysis summary ....................28

B-1. Fort Vancouver National Historic Site vehicle index .......................................................... 31 


\section{ACRONYMS}

BEA Battelle Energy Alliance, LLC

BEV battery electric vehicle

EPA Environmental Protection Agency

EVSE electric vehicle supply equipment

FVNHS Fort Vancouver National Historic Site

GHG greenhouse gas

GSA General Services Administration

GVWR gross vehicle weight rating

ICE internal combustion engine

ITSNA Intertek Testing Services, North America

PEV plug-in electric vehicle (Includes BEVs and PHEVs, but not hybrid electric vehicles)

PHEV plug-in hybrid electric vehicle

VIN vehicle identification number 


\section{AVTA Federal Fleet PEV Readiness Data Logging and Characterization Study for the National Park Service: Fort Vancouver National Historic Site}

\section{INTRODUCTION}

Federal agencies are mandated by the Energy Policy Act of $1992^{2}$, Energy Policy Act of $2005^{3}$,

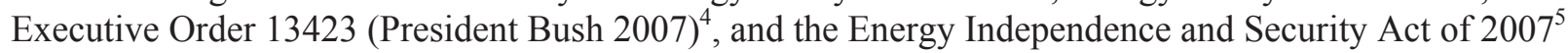
to purchase alternative fuel vehicles, increase consumption of alternative fuels, and reduce petroleum consumption.

Battelle Energy Alliance, LLC (BEA), managing and operating contractor for Idaho National Laboratory, which is the lead laboratory for the U.S. Department of Energy's advanced vehicle testing, manages the Advanced Vehicle Testing Activity Federal Fleet Vehicle Data Logging and Characterization study, which promotes the use of advanced, electric drive vehicle transportation technologies. The Advanced Vehicle Testing Activity focuses its testing activities on emerging and newly commercialized plug-in electric vehicle (PEV) technologies because of the high-energy efficiencies and the ability of PEVs to reduce petroleum consumption. BEA selected Intertek Testing Services, North America (ITSNA) to collect data on federal fleet operations and report the findings on vehicle and mission characterizations to support the successful introduction of PEVs into federal fleets.

Because of the large number of vehicles in the federal fleets within the United States, these fleets provide a substantial opportunity for the introduction of battery electric vehicles (BEVs) and plug-in hybrid electric vehicles (PHEVs). However, to assess the scale of this opportunity, additional data are required to characterize the various missions performed by each fleet and to determine which existing vehicles may be suitable for replacement by a PEV.

Fort Vancouver National Monument was established June 19, 1948, "to preserve as a national monument the site of the original Hudson's Bay stockade (of Fort Vancouver) and sufficient surrounding land to preserve the historical features of the area" for "the benefit of the people of the United States" (62 Stat.352 and the Senate Report on the legislation). A map of Fort Vancouver National Historic Site (FVNHS), shown in Figure 1, covers 210 acres of land and receives more than one million visitors each year. ${ }^{6}$ FVNHS is a part of the Vancouver National Historic Reserve and is an excellent site for fleet evaluation due to its location and its accessibility by the public. To foster its long-term vision outlined in the FVNHS long-range interpretive plan, FVNHS has an opportunity to be a leader in the adoption of BEVs and PHEVs for its fleet.

"For centuries people have recognized the richness of the natural and cultural resources in the area and have conveyed this knowledge to others through oral, written, and scientific records. These explorations and discoveries have influenced and will continue to influence new and changing uses of the area, including impact through settlement and industry on the environment, trade, agriculture, and social organization. Interpretation of this theme will help visitors better understand...Efforts in exploring solutions to today's issues (i.e., public health, environmental preservation, cultural resources preservation, etc.)."7

\footnotetext{
${ }^{2}$ http://thomas.loc.gov/cgi-bin/query/z?c102:h.r.776.enr [accessed January 10, 2014]

${ }^{3}$ http://www.gpo.gov/fdsys/pkg/BILLS-109hr6enr/pdf/BILLS-109hr6enr.pdf [accessed January 10, 2014]

${ }^{4}$ http://www.gsa.gov/portal/content/102452 [accessed January 10, 2014]

${ }^{5}$ http://www.gpo.gov/fdsys/pkg/PLAW-110publ140/pdf/PLAW-110publ140.pdf [accessed January 10, 2014]

${ }^{6}$ http://www.nps.gov/fova/faqs.htm [accessed February 17, 2014]

${ }^{7}$ http://www.nps.gov/fova/parkmgmt/upload/FOVA\%20LRIP\%20-\%20FINAL\%202004.pdf [accessed February 17, 2014]
} 


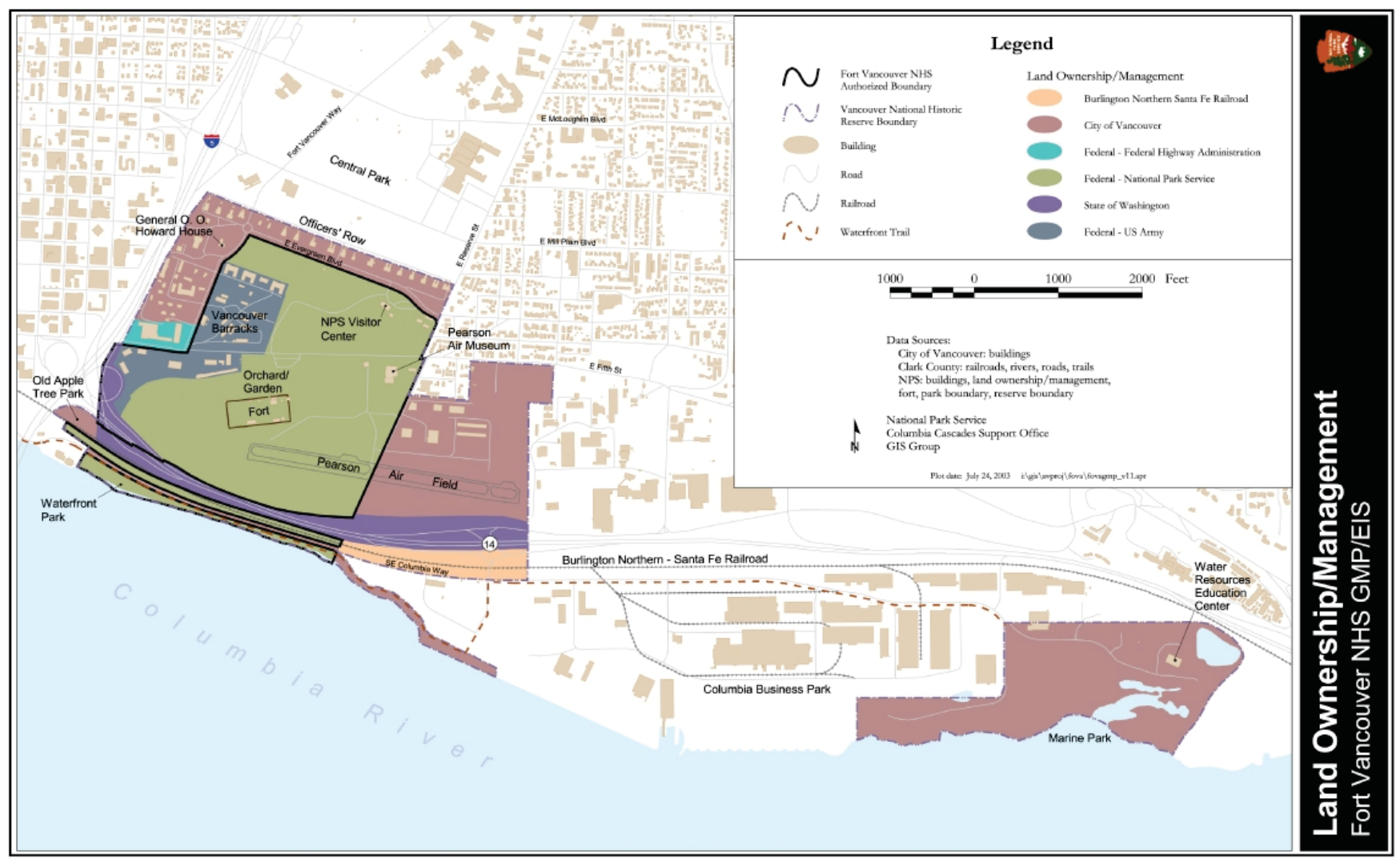

Figure 1. Fort Vancouver National Historic Site.

\section{PROJECT OBJECTIVE}

This study explores federal fleet vehicles and their usage characteristics, with the primary goal of supporting the goals of Presidential Executive Order 13514, which includes the following:

- Pursuing opportunities with vendors and contractors to address and incentivize greenhouse gas (GHG) emission reductions

- Implementing strategies and accommodations for transit, travel, training, and conferences that actively reduce carbon emissions associated with commuting and travel by agency staff

- Meeting GHG emissions reductions associated with other federal government sustainability goals

- Implementing innovative policies and practices that address agency-specific Scope 3 GHG emissions. ${ }^{8}$

Because of the large number of vehicles in the federal fleets, there is a substantial opportunity for PHEV and BEV adoption. Federal fleets offer an opportunity as a first market replacement for alternative fuels due to their scale, refueling patterns, and high vehicle turnover. ${ }^{9}$

This project has the following four defined tasks:

1. Data Collection: Coordinate with the fleet manager to collect data on agency fleet vehicles. This includes collecting fleet vehicle characteristics and installing and monitoring data loggers on select fleet vehicles to characterize their missions.

\footnotetext{
${ }^{8}$ http://energy.gov/sites/prod/files/2013/10/f3/eo13514.pdf [accessed February 5, 2014]

${ }^{9}$ Fleet Purchase Behavior: Decision Processes and Implications for New Vehicle Technologies and Fuel, Nesbitt, Sperling, University of California, Davis 2001
} 
2. Data Analysis and Review: Examine the data collected by the loggers and fleet vehicle characteristics to describe typical fleet activity and incorporate fleet manager input on introducing PEVs into the agency's fleet.

3. PEV Implementation Feedback: Provide feedback to fleet personnel and BEA on the selection criteria and use of PEVs in their specific fleet vehicle missions.

4. Observations and Recommendations: Provide actionable information to implement PEVs into agency fleet operations and assess related impacts for the facility.

Data collected from vehicles include trip distance, idle time, time between uses, and stop locations. Data collection continues for 30 to 60 days using a non-intrusive data logger, which gathers and transmits information using global positioning satellites and cellular service. The data loggers transmit data to the data center at 5 -second intervals when an active signal is present.

Extrapolating the results of this analysis to the larger fleet provides estimates of potential savings in gasoline consumption and GHG emissions. This report also provides recommendations related to fleet management of BEVs and PHEVs for additional consideration.

Fleet managers may use the information supplied in this report to identify which types of vehicles are suitable for BEV or PHEV replacement. BEVs are preferred due to the greater potential reduction of GHG emissions and petroleum usage; however, they may not be suitable for all vehicle missions.

The information in this report supports a final report to BEA/Idaho National Laboratory and the U.S. Department of Energy. The aggregated results for all agencies' fleets will provide an overview of federal fleets, vehicle missions, vehicle use, and agencies' needs to plan and establish a more systematic method for the adoption of BEVs and PHEVs.

\section{METHODS}

\subsection{Fleet Vehicle Survey}

Agency fleet managers selected fleet vehicles for this study and provided basic information for each vehicle, including its managing agency, home base for the vehicle, contact information, primary vehicle mission, vehicle ownership, fuel type, and annual mileage driven. FVNHS provided the information for each vehicle using the form shown in Appendix A.

FVNHS identified three fleet vehicles for inclusion in this study (Table 1). A full inventory of fleet vehicles is unavailable. (Note that Section 4 provides descriptions of the vehicle mission types.)

Table 1. Fleet evaluation.

\begin{tabular}{lcccc}
\hline \multicolumn{1}{c}{ Fleet Evaluation } & & \\
\multicolumn{1}{c}{ Vehicle Mission } & Study & Total Fleet & Mid-Range & Percentage \\
Vehicles & Reported & (Rounded) & Studied \\
\hline $\begin{array}{l}\text { Pool Vehicles } \\
\text { Enforcement Vehicles }\end{array}$ & 1 & & \\
Support Vehicles & 2 & & \\
Transport Vehicles & & & \\
Specialty Vehicles & & & \\
Shuttle/Bus & & & \\
Low Speed Vehicles & & & \\
Total Fleet Vehicles & 3 & & \\
\hline
\end{tabular}


ITSNA coordinated with the FVNHS fleet manager to identify the specific vehicles for data collection for inclusion in the study. The fleet manager assessed their wide range of light-duty vehicles and made selections of high-interest vehicles based on vehicle missions and vehicle type/class. Selection also favored vehicles used at least twice a week. Because data loggers rely on the vehicle's battery power, non-use of the vehicle can result in the vehicle having a depleted battery. ITSNA received no reports of depleted batteries during the study at FVNHS. One support vehicle failed to report data for unknown reasons.

\subsection{Data Collection}

Individual privacy concerns exist when monitoring vehicle movement with data loggers. Data collection occurs by vehicle identification as identified by FVNHS, the data logger number, and the vehicle identification number. ITSNA receives no information related to the vehicle operator and provides no raw data to fleet managers. In this manner, ITSNA does not collect, analyze, or report on individual driving habits.

\subsubsection{Data Logger}

Non-intrusive data loggers, produced by InTouchMVC ${ }^{10}$ and depicted in Figure 2, were placed into the vehicle's onboard diagnostic port to collect and transmit the relevant data. Data logger installation and manual recording of vehicle information typically takes less than 1 minute. Once installed and activated (during vehicle use), the data loggers transmit vehicle information every 5 seconds by cellular communication to the data center.

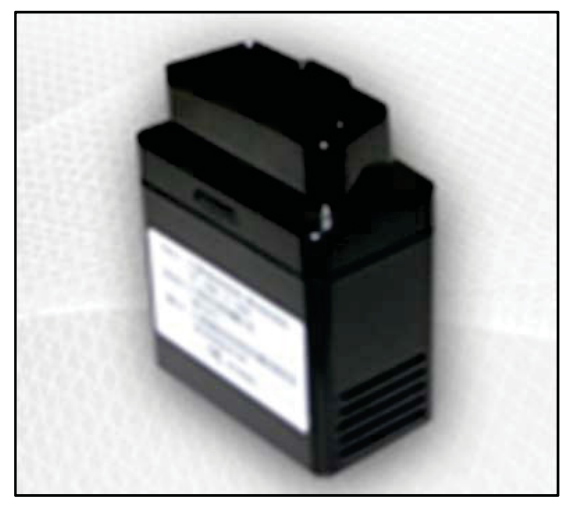

Figure 2. InTouchMVC data logger.

ITSNA maintained the data logger's connectivity and typically verified data transmission weekly. Missing data (reported as "null" values) were frequently the result of lost global positioning system reception, logger device removal, or extended periods in regions with insufficient cellular reception. ITSNA eliminated the vehicle and data logger information if these null values presented a significant impact on the data collected and no resolution was possible. This report also identifies the statistics on this validation process.

The agency removed and shipped the data loggers to ITSNA at the conclusion of the data collection period.

FVNHS requested three data loggers and installed loggers onto three vehicles (i.e., one administrative pool vehicle for the Visitor Center and two support vehicles for maintenance). One support vehicle (i.e., logger 80) provided no data for reasons unknown.

\footnotetext{
${ }^{10}$ www.intouchmvc.com [accessed January 10, 2014]
} 


\subsubsection{Data Captured}

Data consisted of key-on events, key-off events, and position updates logged every minute while the vehicle was keyed-on. The accompanying InTouchMVC fleet reporting software converted these data points into records of trip events, stop events, and idle events.

From these data points, the following information was available for evaluation:

- Trip start and stop time and location

- Trip distance and duration

- Idle start time, location, and duration

- Stop start time, location, and duration.

\subsection{Data Analysis}

\subsubsection{Definitions}

Figure 3 illustrates a vehicle outing that is comprised of trips, stops, and idle events, which may occur over 1 day or several days. The following list provides a definition of these terms:

1. Outing: An outing is the combination of trips and stops that begin at the home base and includes all travel until the vehicle returns home.

2. Trip: A trip begins with a key-on event and ends with the next key-off event.

3. Vehicle stop: A vehicle stop includes both key-off/key-on paired events and idle events.

4. Idle time: Idle time is the amount of time a vehicle spends stationary after a key-on event when the vehicle is not moving for a period of 3 minutes or longer.

5. Trip travel time: Trip travel time is the amount of time required to complete a trip, excluding stops but including idle time.

Definitions of additional analysis and survey terms are as follows:

1. Operating shift: Fleet manager-defined period worked.

2. Study days: Days during which the data loggers are connected.

3. Vehicle days: Study days during which a vehicle is used.

4. Null values: A null value is a data record unusable for analysis for various reasons.

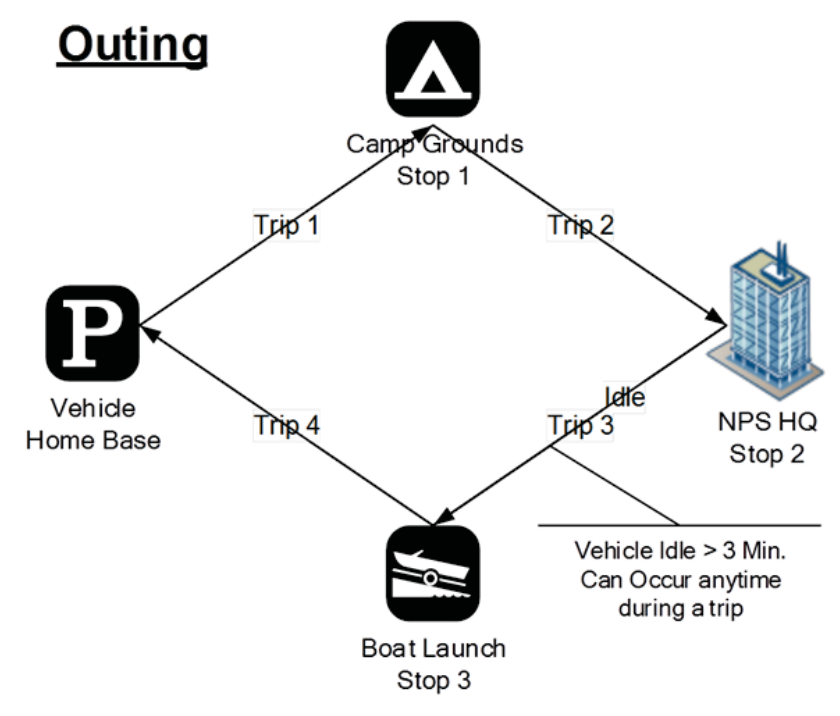

Figure 3. Vehicle outing. 


\subsubsection{Data Evaluation}

Processing the data involved removal of null values and aggregation by different spatial and temporal scales. Aggregation was by day, by trip, and by outing to produce figures showing the patterns of use.

Aggregation by vehicle mission followed to characterize use for the agency fleet. Section 5 presents these results. Data extrapolation occurred to provide overall fleet usage and benefit analysis when fleet information was provided. Section 6 presents these benefits. ITSNA observations are included in Section 7.

Statistical data analysis used Python 2.7, with the Matlab Plotting Library graphics environment (Matplotlib) and spatial display with ESRI ArcGIS. ${ }^{11}$ Frequency distributions summarized the travel behavior of each vehicle and vehicle mission during the study period. Rounding of values in the tables and figures is to three significant digits.

\section{VEHICLES}

\subsection{Vehicle Missions}

The vehicle mission is an important characteristic in the fleet study. Information used to define the vehicle mission includes the vehicle's configuration, vehicle use, classification per 40 CFR Part 600.31582, the participating agency use and general vehicle use. ITSNA has found that fleet vehicles generally fall into seven mission/vehicle categories that are listed as follows and are depicted in Figure 4:

1. Pool vehicles: A pool vehicle is any automobile (other than low-speed vehicles identified below) manufactured primarily for use in passenger transportation, with not more than 10 passengers.

2. Enforcement vehicles: Vehicles specifically approved in an agency's appropriation act for use in apprehension, surveillance, police, or other law enforcement work. This category also includes site security vehicles, parking enforcement, and general use, but the vehicles are capable of requirements to support enforcement activities. Appendix C provides further definition.

3. Support vehicles: Vehicles assigned to a specific work function or group to support the mission of that group. Vehicles are generally passenger vehicles or light-duty pickup trucks and may contain after-market modifications to support the mission.

4. Transport vehicles: Light, medium, or heavy-duty trucks used to transport an operator and tools or equipment of a non-specific design or nature. The vehicle's uses include repair, maintenance or delivery.

5. Specialty vehicles: Vehicles designed to accommodate a specific purpose or mission (such as ambulances, mobile cranes, and handicap controls).

6. Shuttles/buses: Vehicles designed to carry more than 12 passengers and further outlined in 49 CFR 532.2.

7. Low-speed vehicle: Vehicles that are legally limited to roads with posted speed limits up to $45 \mathrm{mph}$ and that have a limited load-carrying capability.

\subsection{Alternative Fuel Vehicles}

As the operating agency, FVNHS has a unique opportunity to plan for the adoption of BEVs and PHEVs, along with planning for the supporting infrastructure. The adoption of PHEVs and BEVs is a primary goal of General Services Administration (GSA) and supports the directives previously referenced.

As GSA increases its certification of PHEVs and BEVs, agencies can plan for vehicle replacement through GSA for passenger vehicles and trucks. Table 2 presents the replacement requirements for fleet vehicles.

\footnotetext{
${ }^{11}$ www.esri.com [accessed January 10, 2014]
} 


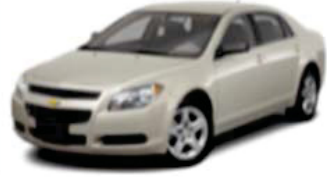

Pool Vehicle

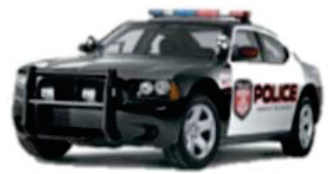

Enforcement Vehicle

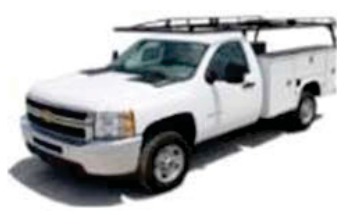

Support Vehicle

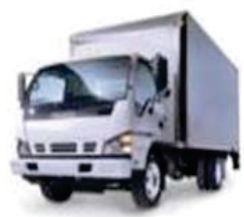

Transport Vehicle

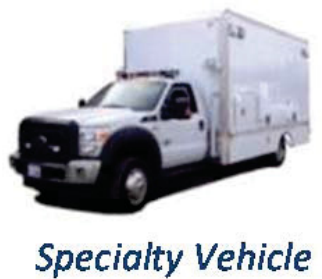

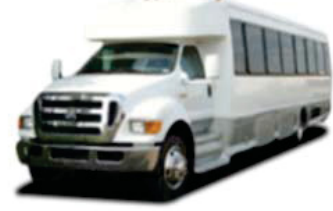

Shuttle / Bus

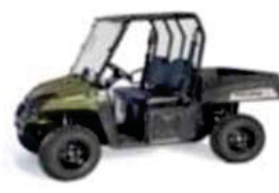

Low Speed

Vehicles

Figure 4. Vehicle missions and example vehicles.

Table 2. General Services Administration vehicle replacement requirements.

\begin{tabular}{lccc}
\hline \multicolumn{3}{c}{ GSA Vehicle Replacement Requirements ${ }^{12}$} & \\
& Fuel Type & Years & Miles \\
\hline Passenger vehicles & Gasoline or AFV & 3 and & 36,000 \\
& & 4 and & 24,000 \\
& & 5 and & Any miles \\
& & Any year and & 75,000 \\
& Hybrid & 5 and & Any miles \\
Light trucks $4 \times 2$ & LSEV & 6 and & Any miles \\
& Non-diesel & 7 or & 65,000 \\
& Diesel & 8 or & 150,000 \\
Light trucks $4 \times 4$ & Hybrid & 7 and & Any miles \\
& Non-diesel & 7 or & 60,000 \\
& Diesel & 8 or & 150,000 \\
& Hybrid & 7 and & Any miles \\
\hline
\end{tabular}

\subsection{Battery Electric Vehicle/Plug-in Hybrid Electric Vehicle Benefits/Challenges}

BEVs are powered $100 \%$ by the battery energy storage system onboard the vehicle. The Nissan LEAF is an example of a BEV. Because the BEV has no other energy source for propulsion, the range, power requirements, and mission of the needed vehicle factor greatly in purchasing decisions.

Maximizing BEV capabilities typically requires batteries more than an order of magnitude larger than the batteries in hybrid electric vehicles.

PHEVs obtain their power by two energy sources. The typical PHEV configuration utilizes a battery and an internal combustion engine (ICE), powered by either gasoline or diesel. PHEV designs differ between manufacturers, although all have a charge-depleting mode in which the off-board energy supplied to the battery discharges to propel the vehicle, and a charge-sustaining mode, in which the battery assists the ICE; however, the charge-sustaining mode provides the majority of the propulsion power and the state of charge is maintained between set limits. Some PHEVs exhibit completely

\footnotetext{
${ }^{12}$ http://www.gsa.gov/graphics/fas/VehicleReplacementStandardsJune2011Redux.pdf [accessed January 10, 2014]
} 
all-electric charge-depleting modes, while others have a blended charge-depleting mode in which both the battery and the ICE provide propulsion power while the battery depletes.

\subsubsection{Battery Electric Vehicle/Electric Vehicle Benefits/Challenges ${ }^{13}$} BEVs:

The U.S. Environmental Protection Agency (EPA) identifies the following benefits/challenges of

- Energy efficient: Electric vehicles convert about 59 to $62 \%$ of the electrical energy from the grid to power at the wheels, whereas conventional gasoline vehicles only convert about 17 to $21 \%$ of the energy stored in gasoline to power at the wheels.

- Environmentally friendly: PEVs emit no tailpipe pollutants, although the power plant producing the electricity may emit them. Electricity from nuclear, hydro, solar, or wind-powered plants causes no air pollutants.

- Performance benefits: Electric motors provide quiet, smooth operation and exhibit maximum torque at zero and low speeds, while also requiring less maintenance than ICEs.

- Reduce energy dependence: Electricity is a domestic energy source.

The EPA also identifies challenges associated with BEVs, including the following:

- Driving range: Most BEVs can only travel about 100 to 200 miles (or less) before recharging, whereas gasoline vehicles can often travel over 300 miles before refueling and some much further.

- Recharge time: Fully recharging the battery pack can take 4 to 8 hours. Even a "quick charge" to $80 \%$ capacity can take 30 minutes.

- Battery cost: The large battery packs are expensive and may need to be replaced one or more times.

- Bulk and weight: Battery packs are heavy and take up considerable vehicle space.

\subsubsection{Plug-in Hybrid Electric Vehicle Benefits/Challenges ${ }^{14}$}

The U.S. Environmental Protection Agency (EPA) identifies the following benefits/challenges of PHEVs:

- Less petroleum use: PHEVs are expected to use about 40 to $60 \%$ less petroleum than conventional vehicles. Because electricity is produced primarily from domestic resources, PHEVs reduce dependence on oil.

- Fewer GHG emissions: PHEVs are expected to emit fewer GHG emissions than conventional vehicles, but as with BEVs, the difference depends largely on the type of power plant supplying the electricity.

- Higher vehicle costs, lower fuel costs: PHEVs will likely cost $\$ 1,000$ to $\$ 7,000$ more than comparable non-PHEVs. Fuel will cost less since electricity is much cheaper than gasoline, but the fuel savings depends on how much driving is done on off-board electrical energy.

- Recharging takes time: Recharging the battery typically takes several hours. However, PHEVs do not have to be plugged in to be driven. They can be fueled solely with gasoline, but will not achieve maximum range, fuel economy, or fuel savings without charging.

- Measuring fuel economy: Because a PHEV can operate on electricity alone, gasoline alone, or a mixture of the two, EPA provides a fuel economy estimate for gasoline-only operation (charge-sustaining mode), electric-only operation (all-electric charge-depleting mode), or combined gasoline and electric operation (blended charge-depleting mode).

\footnotetext{
${ }^{13}$ http://www.fueleconomy.gov/feg/evtech.shtml [accessed 27 December 2013]

${ }^{14}$ http://www.fueleconomy.gov/feg/phevtech.shtml [accessed 19 July 2013]
} 
In many cases, the PEV retail cost is higher than a non-PEV model. This incremental purchase cost may be a challenge; however, many original equipment manufacturers have offered incentives to encourage the use and adoption of BEVs and PHEVs. Some original equipment manufacturers have recently reduced the vehicle cost, while also increasing vehicle range. Additionally, federal and state incentives have increased the attractiveness of purchasing a PEV. A common assumption is that increasing PEV sales will result in a decrease in the incremental purchase cost and a positive feedback loop will ensue.

\subsection{Plug-In Electric Vehicle Availability}

GSA provides a summary of light and medium-duty passenger vehicles available for lease or purchase through the GSA portal ${ }^{15}$, although not all BEVs and PHEVs currently on the market are 'certified' to be GSA replacements. Vehicles not on the GSA list of 'certified' vehicles require an agency to self-certify a functional need or alternative measures for exemptions. Table 3 summarizes the vehicles that may be suitable replacements and are certified replacements through GSA. The Nissan Leaf, Ford Transit Connect, and Mitsubishi i-MiEV are not included in the alternative fuel guide for 2014, but they have appeared in previous guides.

Replacement is dependent on vehicle configuration characteristics and the vehicle mission. Further evaluation related to vehicle purpose, mission, and need should be completed.

Tables 4 through 7 provide summaries of PHEVs and BEVs either currently available or near commercialization in both passenger cars and pickup trucks, but do not appear on the GSA 'certified' vehicle list. These vehicles may qualify for use by the agency through demonstrating a functional need.

Table 3. General Services Administration-certified battery electric vehicles/plug-in electric vehicles for 2014.

\begin{tabular}{lcccc}
\hline \multicolumn{1}{c}{ Make/Model } & GSA Class & Type & City/Highway & GSA Incremental Price \\
\hline Chevrolet Volt & Sedan, Compact & PHEV & $101 / 93 \mathrm{MPGe}$ & $\$ 17,087.18$ \\
Ford C-MAX PHEV & Sedan, Midsize & PHEV & $108 / 92 \mathrm{MPGe}$ & $\$ 14,899.52$ \\
Ford Focus BEV & Sedan, Compact & BEV & $110 / 99 \mathrm{MPGe}$ & $\$ 16,573.09$ \\
Ford Fusion Energi & Sedan, Midsize & PHEV & $108 / 92 \mathrm{MPGe}$ & $\$ 19,289.99$ \\
\hline
\end{tabular}

Table 4. Original equipment manufacturer plug-in hybrid electric vehicle cars and availability.

\begin{tabular}{lcc}
\multicolumn{1}{c}{ Make } & Model & $\begin{array}{c}\text { Model Year/Estimated Date } \\
\text { for Commercialization }\end{array}$ \\
\hline Audi & A3 eTron PHEV & 2015 \\
BMW & i8 & 2015 \\
Cadillac & ELR & 2014 \\
Chevrolet & Volt & 2011 \\
Honda & Accord PHEV & 2013 \\
Mercedes & S-Class PHEV & 2015 \\
Porsche & Panamera PHEV & 2013 \\
Toyota & Prius PHEV & 2012 \\
Volvo & V60 Plug-in & 2016 \\
\hline
\end{tabular}

${ }^{15}$ http://www.gsa.gov/portal/content/104224 [accessed March 6, 2014] 
Table 5. Original equipment manufacturer battery electric vehicle cars and availability.

\begin{tabular}{lcc}
\hline \multicolumn{1}{c}{ Make } & Model & $\begin{array}{c}\text { Model Year/Estimated Date } \\
\text { for Commercialization }\end{array}$ \\
\hline BMW & i3 (Megacity) & 2014 \\
BMW & i3 & 2014 \\
Chevrolet & Spark & 2013 \\
Fiat & 500e & 2013 \\
Ford & Focus electric & 2012 \\
Honda & Fit EV & 2013 \\
Kia & Soul EV & 2015 \\
Mercedes & B-Class E-Cell & 2015 \\
Mitsubishi & i-MiEV & 2012 \\
Nissan & LEAF & 2011 \\
Scion & IQ EV & 2016 \\
Smart & ED & 2013 \\
Tesla & Model S & 2012 \\
Tesla & Model X & 2015 \\
Volkswagen & Golf Blue-e-Motion & 2014 \\
Volvo & C30 Electric & 2015 \\
\hline
\end{tabular}

Table 6. Original equipment manufacturer plug-in hybrid electric vehicle trucks and vans and availability.

\begin{tabular}{lcc}
\multicolumn{1}{c}{ Make } & Model & $\begin{array}{c}\text { Model Year/Estimated Date } \\
\text { for Commercialization }\end{array}$ \\
\hline Land Rover & Range Rover Sport & 2016 \\
Mitsubishi & Outlander PHEV & 2015 \\
Via & VR300 & 2013 \\
\hline
\end{tabular}

Table 7. Original equipment manufacturer battery electric vehicle trucks and vans and availability.

\begin{tabular}{lcc} 
Make & Model & $\begin{array}{c}\text { Model Year/Estimated Date } \\
\text { for Commercialization }\end{array}$ \\
\hline Nissan & eNV200 & 2015 \\
Toyota & RAV4 EV & 2014 \\
\hline
\end{tabular}

\subsection{Plug-In Electric Vehicle Charging}

Refueling electric vehicles presents some challenges not encountered when refueling petroleum-fueled vehicles. Recharging the battery of a PHEV follows the same methodology as that for BEVs. This section provides basic information on recharging PEVs.

\subsubsection{Electric Vehicle Supply Equipment Design}

4.5.1.1 Charging Components. PEV charging stations, more correctly identified as Electric Vehicle Supply Equipment (EVSE), deliver electric power from the utility to the applicable charge port on the vehicle. Figure 5 illustrates the primary components of a typical EVSE, which in Figure 5 is an alternating current (AC) Level 2. 


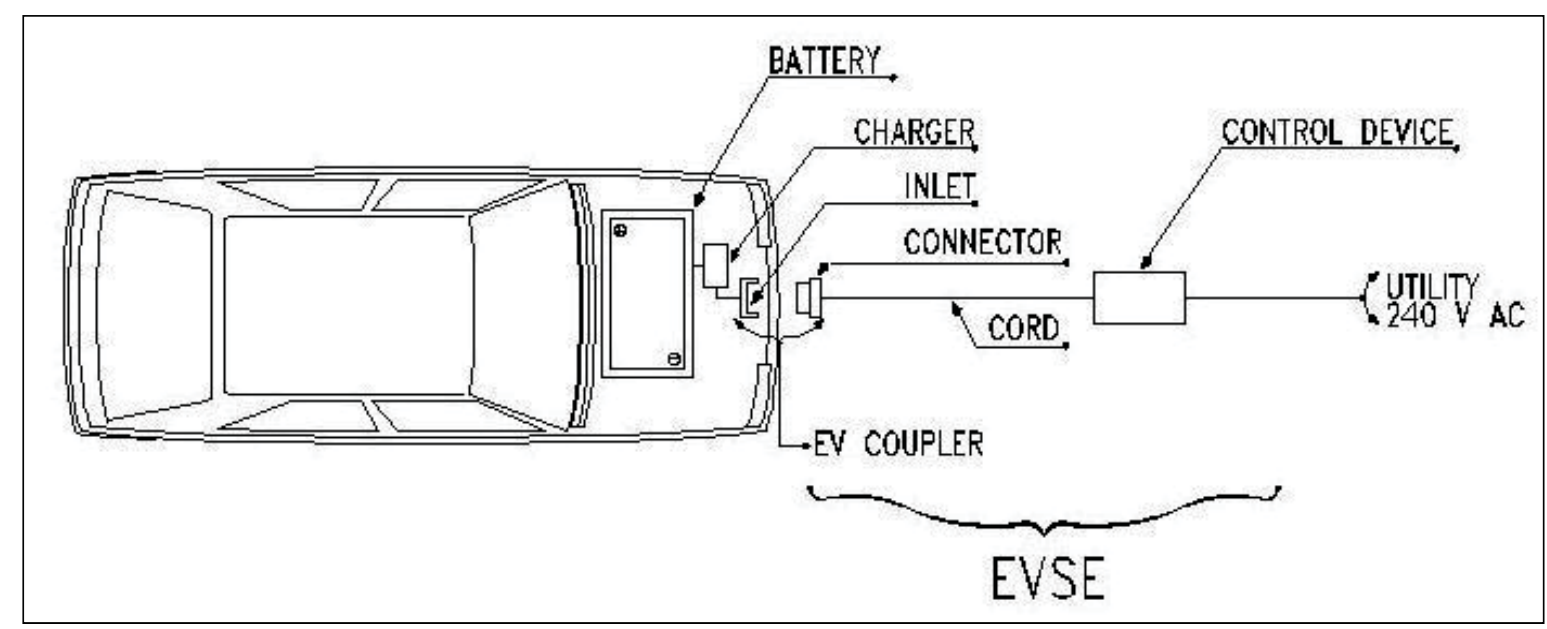

Figure 5. Alternating Current Level 2 charging diagram. ${ }^{16}$

The electric utility delivers AC current to the charging location. The conversion from AC to direct current (DC) electricity necessary for battery charging can occur either on or off board the vehicle. Section 4.5.1.2 provides further explanation of the different EVSE configurations. For onboard conversion, AC current flows through the PEV inlet to the onboard charger. The charger converts AC to the DC current required to charge the battery. A connector attached to the EVSE inserts into a PEV inlet to establish an electrical connection to the PEV for charging and information/data exchange. Off-board conversion, also known as DC charging, proceeds in a similar manner except that the AC to DC conversion occurs in a charger that is off-board the vehicle and, thus, bypasses any onboard charger. For both AC and DC charging, the PEV's battery management system on board the vehicle controls the battery rate of charge, among other functions. All current PEVs have an onboard charger; some BEVs (but no PHEVs currently) accommodate DC charging.

4.5.1.2 Charging Configurations and Ratings. The Society of Automotive Engineers standardized the requirements, configurations, and equipment followed by most PEV suppliers in the United States in the J1772 Standard. Figure 6 summarizes these attributes and the estimated recharge times. Actual recharge times depend on the onboard equipment including the charger, battery, and battery management system.

Most PEV manufacturers supply an AC Level 1 cord set with the vehicle, which provides sufficient capabilities for some drivers, but more typically provide an emergency backup capability because of the long recharge times. AC recharging capabilities found in public more typically are AC Level 2 . Figure 7 identifies a typical J1772-compliant inlet and connector for both AC Levels 1 and 2.

The J1772 standard also identifies requirements for DC charging. For PEVs that accept both AC and DC inputs, the Society of Automotive Engineers approved a single connector and inlet design. Figure 8 shows this connector, which is called the J1772 combo connector.

Some PEVs delivered in the United States prior to the approval of the J1772 standard for DC charging employ the Japanese-based CHAdeMO standard for connector and inlet design. Figure 9 shows this connector.

The presence of the two separate standards for DC charging presents challenges for vehicle owners to ensure that the EVSE accessed provides the appropriate connector for their vehicle inlet. Not all PEV suppliers include DC charging options. BEV suppliers more typically provide DC inlets than PHEV suppliers, because the rapid recharging provides opportunities for expanded vehicle range with minimal operator wait times. PHEV operators can rely on the gasoline drive in the event they deplete the vehicle's battery; at present, no PHEV on the market or near commercialization has DC charging capability

\footnotetext{
${ }^{16}$ http://avt.inel.gov/pdf/EVProj/EVChrgInfraDeployGuidelinesPhoenixVer3.2.pdf [accessed April 10, 2014]
} 
(although the Mitsubishi Outlander PHEV is rumored to be offering DC charging capability as an option). It is noted that DC Level 1 and DC Level 2 charging are commonly combined and labeled "DC fast charging."

\begin{tabular}{|c|c|c|c|}
\hline \multicolumn{4}{|c|}{ SAE Charging Configurations and Ratings Terminology } \\
\hline \multirow{5}{*}{$\begin{array}{l}\text { AC level } 1 \\
(\text { SAE J1772 }\end{array}$} & PEV includes on-board charger & \multirow[t]{5}{*}{${ }^{*}$ DC Level 1} & EVSE includes an off-board charger \\
\hline & $\begin{array}{l}120 \mathrm{~V}, 1.4 \mathrm{~kW} @ 12 \mathrm{amp} \\
120 \mathrm{~V}, 1.9 \mathrm{~kW} @ 16 \mathrm{amp}\end{array}$ & & $200-450 \mathrm{~V} \mathrm{DC}$, up to $36 \mathrm{~kW}(80 \mathrm{~A})$ \\
\hline & Est. charge time: & & Est. charge time ( $20 \mathrm{~kW}$ off-board charger): \\
\hline & PHEV: 7 hrs (SOC* $-0 \%$ to full) & & PHEV: $22 \mathrm{~min}$. (SOC* $-0 \%$ to $80 \%$ ) \\
\hline & BEV: $17 \mathrm{hrs}$ (SOC $-20 \%$ to full) & & BEV: 1.2 hrs. (SOC $-20 \%$ to $100 \%)$ \\
\hline \multirow[t]{11}{*}{$\begin{array}{l}\text { AC level } 2 \\
\text { (SAE J1772 }\end{array}$} & $\begin{array}{l}\text { PEV includes on-board charger (see below for different } \\
\text { types) }\end{array}$ & \multirow[t]{6}{*}{ *DC Level 2} & EVSE includes an off-board charger \\
\hline & $240 \mathrm{~V}$, up to $19.2 \mathrm{~kW}(80 \mathrm{~A})$ & & $200-450 \mathrm{~V} \mathrm{DC}$, up to $90 \mathrm{~kW}(200 \mathrm{~A})$ \\
\hline & Est. charge time for $3.3 \mathrm{~kW}$ on-board charger & & Est. charge time ( $45 \mathrm{~kW}$ off-board charger): \\
\hline & PEV: 3 hrs (SOC* - $0 \%$ to full) & & PHEV: $10 \mathrm{~min}$. (SOC* $-0 \%$ to $80 \%$ ) \\
\hline & BEV: $7 \mathrm{hrs}$ (SOC $-20 \%$ to full) & & BEV: $20 \mathrm{~min}$. (SOC $-20 \%$ to $80 \%$ ) \\
\hline & Est. charge time for $7 \mathrm{~kW}$ on-board charger & & \\
\hline & PEV: 1.5 hrs (SOC* - 0\% to full) & \multirow[t]{6}{*}{ *DC Level 3 (TBD) } & EVSE includes an off-board charger \\
\hline & BEV: 3.5 hrs (SOC $-20 \%$ to full) & & $200-600 \mathrm{~V} \mathrm{DC}$ (proposed) up to $240 \mathrm{~kW}(400 \mathrm{~A})$ \\
\hline & Est. charge time for $20 \mathrm{~kW}$ on-board charger & & Est. charge time ( $45 \mathrm{~kW}$ off-board charger): \\
\hline & PEV: $22 \mathrm{~min}$. (SOC $-0 \%$ to full) & & BEV (only): $<10 \mathrm{~min}$. (SOC* $-0 \%$ to $80 \%$ ) \\
\hline & BEV: $1.2 \mathrm{hrs}$ (SOC $-20 \%$ to full) & & \\
\hline •AC Level 3 (TBD) & $>20 \mathrm{~kW}$, single phase and 3 phase & & \\
\hline \multicolumn{4}{|c|}{$\begin{array}{l}\text { "Not finalized } \\
\text { Voltages are nominal configuration voltages, not coupler ratings } \\
\text { Rated Power is at nominal configuration operating voltage and coupler rated current } \\
\text { Ideal charge times assume } 90 \% \text { efficient chargers, } 150 \mathrm{~W} \text { to } 12 \mathrm{~V} \text { loads and no balancing of Traction Battery Pack }\end{array}$} \\
\hline \multicolumn{4}{|c|}{$\begin{array}{l}\text { 1) BEV ( } 25 \mathrm{kWh} \text { usable pack size) charging always starts at } 20 \% \mathrm{SOC} \text {, faster than a } 1 \mathrm{C} \text { rate (total capacity charged in one hour) will also stop at } 80 \% \text { SOC instead of } \\
100 \% \\
\begin{array}{ll}\text { 2) PHEV can start from } 0 \% \text { SOC since the hybrid mode is available. } & \text { Developed by the SAE Hybrid Committee }\end{array}\end{array}$} \\
\hline
\end{tabular}

Figure 6. Society of Automotive Engineers charging configurations and ratings terminology. ${ }^{17}$

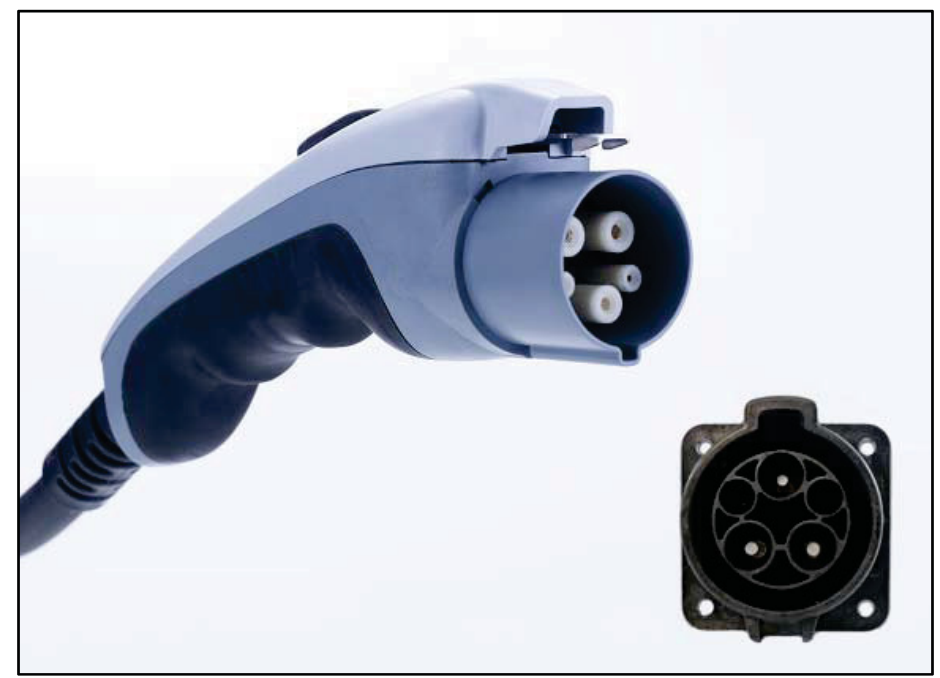

Figure 7. J1772 connector and inlet. ${ }^{18}$

${ }^{17}$ http://www.sae.org/smartgrid/chargingspeeds.pdf [accessed January 15, 2014]

${ }^{18}$ http://carstations.com/types/j09 [accessed January 15, 2014] 

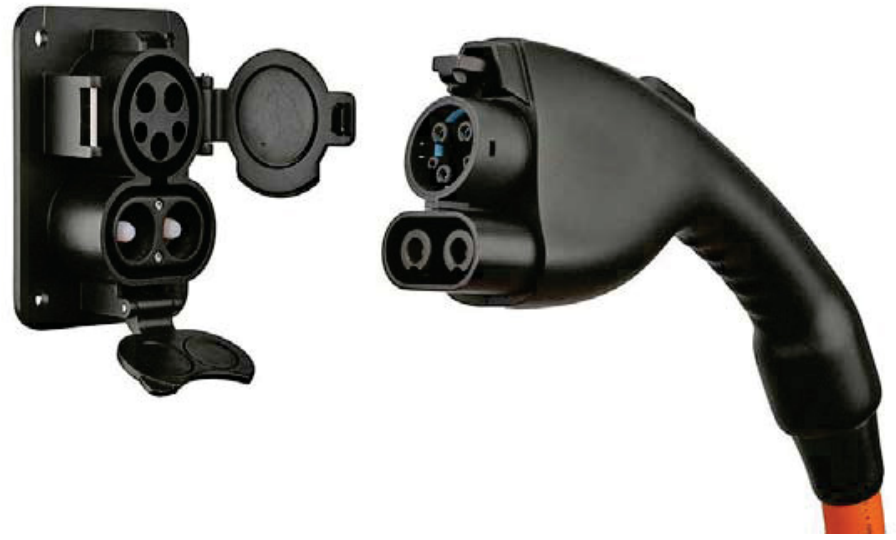

Figure 8. J1772-compliant combo connector. ${ }^{19}$

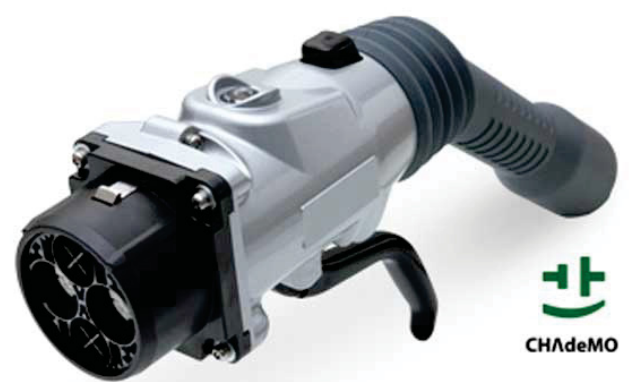

Figure 9. CHAdeMO-compliant connector. ${ }^{20}$

Because the battery of a BEV is typically much larger than that of a PHEV, recharge times are longer (see Figure 6). BEVs that see daily mileage near the limits of the advertised range do better when recharged using AC Level 2 EVSE or DC fast charging, because AC Level 1 recharge times are usually extensive. PHEVs, on the other hand, generally can use AC Level 1 EVSE for overnight charging to ensure a fully charged battery at the start of daily use. AC Level 2 EVSE units provide greater range in the shortest amount of time when intermediate or opportunity charging. DC fast charging provides the fastest recharge capability for those vehicles equipped with DC fast charge inlets.

\subsubsection{Electric Vehicle Supply Equipment Stations}

AC Level 2 charging is the predominant rating of publicly accessible EVSE because of its wide acceptance by auto manufacturers and faster than AC Level 1 recharge times. Purchase and installation costs are more manageable than DC fast chargers and less space is required. There are several manufacturers of AC Level 2 equipment and the agency should review brands for comparison purposes. Figure 10 provides an example of a public AC Level $2 \mathrm{EVSE}^{21}$.

DC fast chargers also are available from several manufacturers. Figure 11 illustrates one such charger. ${ }^{22}$ This particular charger uses the CHAdeMO connector standard.

In general, installation costs are higher for the DC fast charger because of the higher voltage requirements and the inclusion of the $\mathrm{AC}$ to $\mathrm{DC}$ charger and other safety and design features. Costs for

\footnotetext{
${ }^{19}$ http://www.zemotoring.com/news/2012/10/sae-standardizes-j1772-fast-dc-charging-up-to-100-kw [accessed January 15, 2014]

${ }^{20} \mathrm{https} / / /$ radio.azpm.org/p/azspot/2012/5/10/1632-electric-cars/ [accessed January 15, 2014]

${ }^{21}$ www.eaton.com/ [accessed January 29, 2014]

22 http://nissanqc.com/ \{accessed March 14, 2014]
} 
both types are highly dependent on site characteristics such as distance to the nearest power source, asphalt or concrete cutting and repair, conduit requirements and payment systems, if any.

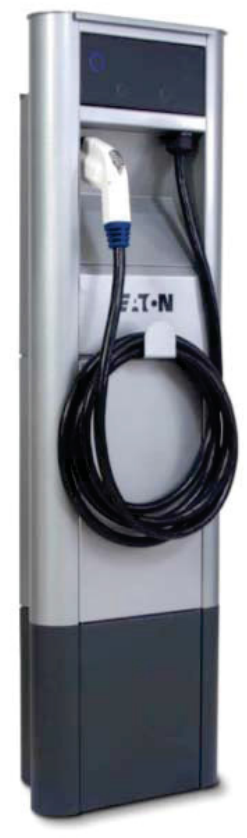

Figure 10. Alternating current Level 2 electric vehicle supply equipment.

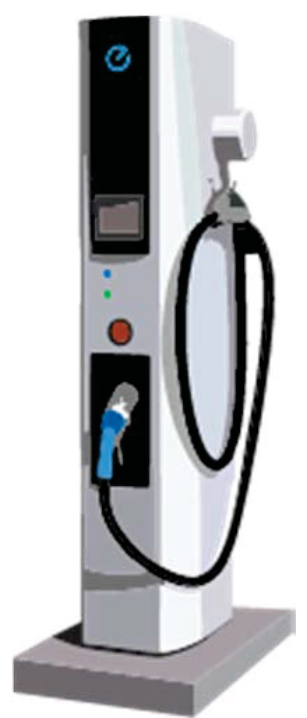

Figure 11. Direct current fast charger.

Payment and equipment control systems included by some suppliers provide the potential for use by privately owned vehicles for a fee, but allow agency fleet vehicle use without direct payment. These systems allow for accurate record keeping of vehicle charging requirements.

\section{FORT VANCOUVER NATIONAL HISTORIC SITE ANALYSIS 5.1 Survey Results}

Three vehicles were included in the study at FVNHS. One is a pool vehicle located primarily at the visitor center (minivan) and two are support vehicles (pickup trucks). One support vehicle failed to provide data. Table 8 presents a summary of these vehicles. A report of all fleet vehicles onsite at FVNHS is not available. 
Table 8. Vehicle survey summary.

\begin{tabular}{lcccc}
\multicolumn{1}{c}{ Mission } & Minivan & Truck & Sedans & Total \\
\hline Pool Vehicle & 1 & & & 1 \\
Support Vehicle & & 2 & & 2 \\
Other Vehicles & & & \\
Total & 1 & 2 & 3 \\
\hline
\end{tabular}

The missions of these two categories vary considerably; therefore, analysis is by mission rather than a combination of vehicles.

\subsection{Data Validity}

FVNHS data collection took place from February 12, 2013, through March 31, 2013 (47 calendar days). The following sections detail the data for collected each vehicle.

Of the data collected, $99.5 \%$ was suitable for inclusion in the analysis, while null values exist for the balance. Table 9 shows this information by mission type. As noted previously, one support vehicle provided no data.

Table 9. Vehicle data logger reporting summary.

\begin{tabular}{lccc}
\multicolumn{4}{c}{ Vehicle Data Logger Reporting Summary } \\
Mission & \% Collected & \% Null Values & Total \\
\hline Pool Vehicle & 99.8 & 0.2 & $100 \%$ \\
Support Vehicle & 99.5 & 0.5 & $100 \%$ \\
All Vehicles & 99.5 & 0.5 & $100 \%$ \\
\hline
\end{tabular}

\subsection{Pool Vehicle Evaluation}

\subsubsection{Survey and Site Information}

Pool vehicles typically are light-duty motor vehicles and primarily are used in passenger transportation, with not more than 10 passengers. Pool missions can vary by agency, location, and jurisdiction; however, they typically are sedans, minivans, vans, or small pickup trucks. The test data show that incorporation of BEVs and/or PHEVs into the pool mission is a definite possibility. The total number of pool vehicles in the fleet was unreported, but the analysis for this one vehicle shows PHEV capabilities are required.

\subsubsection{Pool Vehicle}

This section details the data available for the pool vehicle (see Tables 10 and 11).

Table 10. Pool vehicle details.

\begin{tabular}{|c|c|c|}
\hline \multirow{5}{*}{$\theta^{2}=-8=$} & Make/Model/Year & Chevrolet/Uplander/2007 \\
\hline & EPA Class Size & Minivan \\
\hline & Mission & Pool \\
\hline & Parking Location & Visitor Center \\
\hline & Fleet Vehicle ID & G41-4545F \\
\hline \multirow{5}{*}{ www.edmunds.com } & Fuel Type & Gas E85 \\
\hline & EPA Label/MPG (City/Hwy/Combined) & $16 / 23 / 1912 / 17 / 14$ \\
\hline & EPA GHG Emissions (Grams $\left.\mathrm{CO}_{2} / \mathrm{Mi}\right)$ & 468450 \\
\hline & Study ID & Logger 77 \\
\hline & Total Vehicle Days/Total Study Days & $19 / 46$ \\
\hline
\end{tabular}


Logger 77 collected data on this vehicle for a period of 46 study days, during which 19 days showed usage. Data validation occurred on $99.2 \%$ of the vehicle data, while $0.8 \%$ of the data were null values. The vehicle's primary home base is located at the Visitor Center.

Table 11. Pool vehicle travel summary.

\begin{tabular}{lcccc}
\hline \multicolumn{5}{c}{ Vehicle G41-4545F Travel Summary } \\
\\
Per Day & Per Outing & Per Trip & \\
& Average/Peak & Average/Peak & Average/Peak & Total \\
\hline Travel Distance (Miles) & $39.3 / 213.7$ & $20.7 / 277.8$ & $5.97 / 86.7$ & 746 \\
Travel Time (Minutes) & $73.6 / 291$ & $38.9 / 414$ & $11.2 / 106$ & 1,400 \\
Idle Time (Minutes) & $4.7 / \mathrm{NA}$ & $2.5 / \mathrm{NA}$ & $0.7 / \mathrm{NA}$ & 90 \\
\hline
\end{tabular}

During the study period, the vehicle traveled a total distance of 746 miles and logged 24 hours of driving usage. Figure 12 shows the travel summary for the pool vehicle by daily mileage and daily usage time.

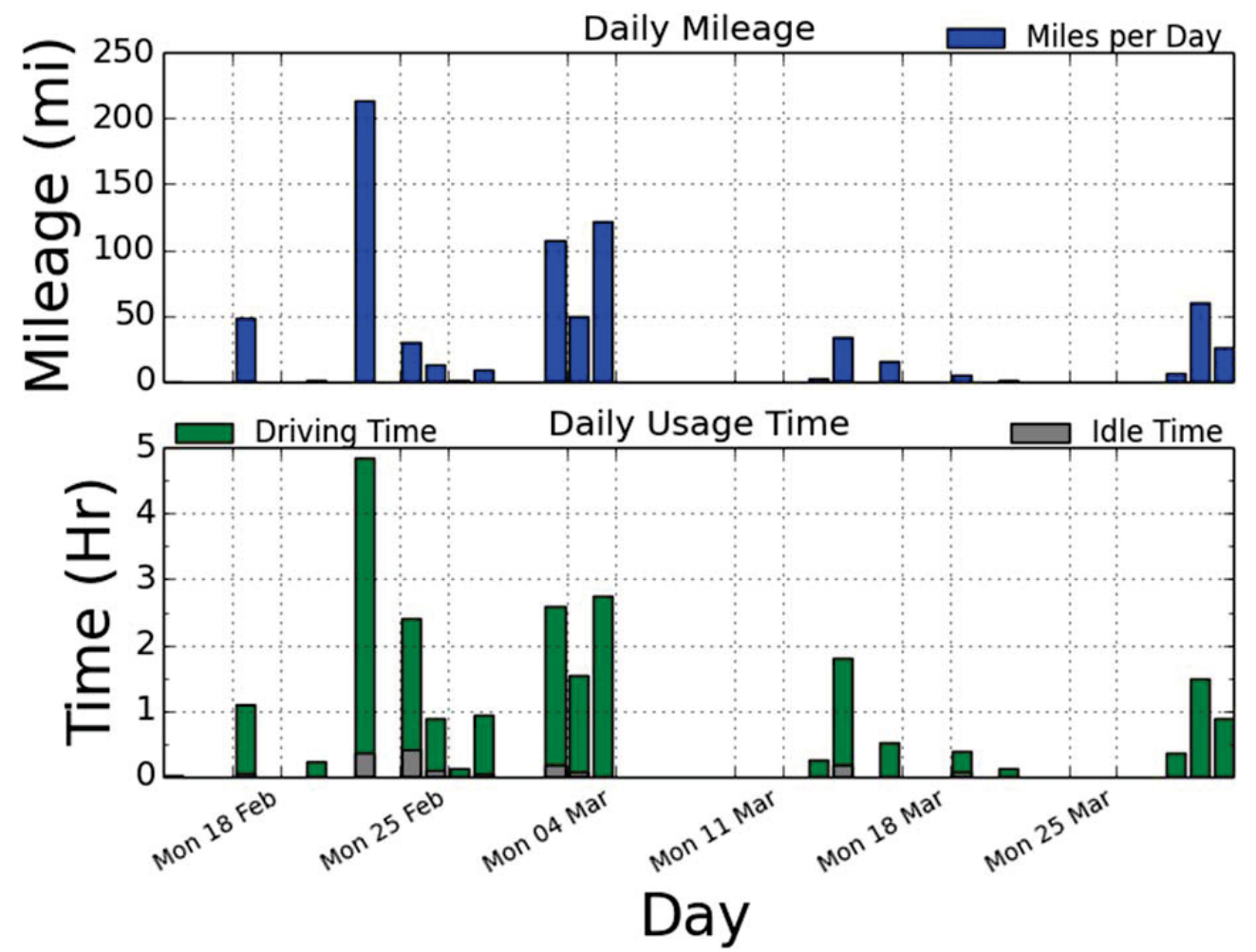

Figure 12. Daily mileage and time for the pool vehicle.

Figure 13 identifies the hours of the day that the vehicle was typically in use.

Stop information identifies where the vehicle parked for a significant time. EVSE, if placed at highly utilized stop locations, provides additional charging opportunities for BEVs. Table 12 shows the stop distances and duration for the pool vehicle and Figure 14 shows the most frequented stop locations. 


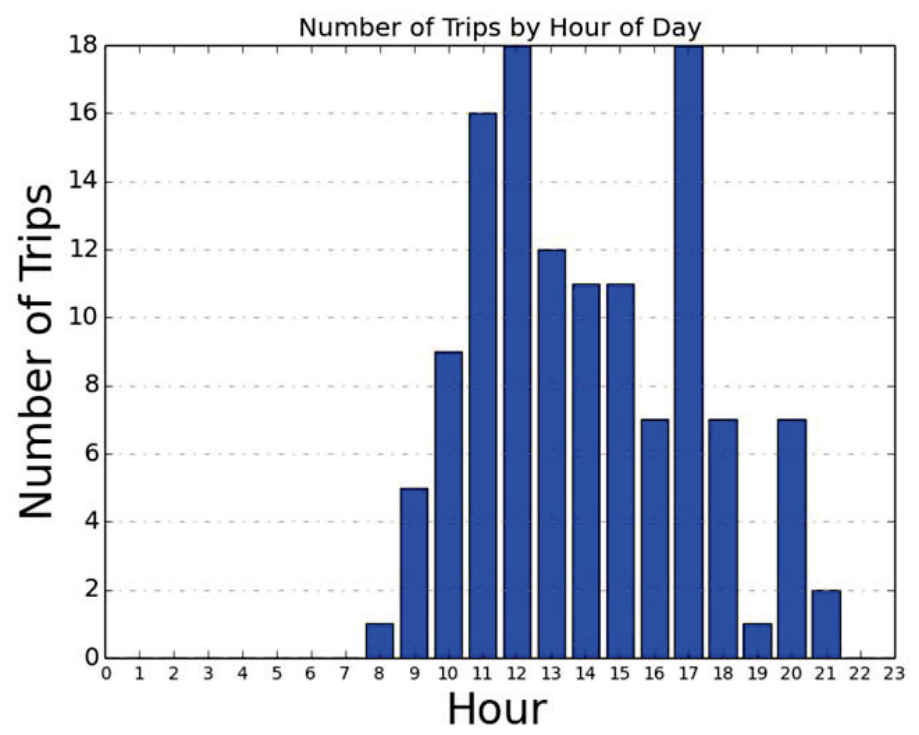

Figure 13. Pool vehicle daily usage time.

Table 12. Pool vehicle stop information.

\begin{tabular}{ccccc}
\hline \multicolumn{2}{c}{ Stop Distance from Home Base } & \multicolumn{2}{c}{ Stop Duration } \\
$\begin{array}{c}\text { Distance From } \\
\text { Home Base (Miles) }\end{array}$ & Stops & Percentage of Total & Stop Duration (Hours) & $\begin{array}{c}\text { Percentage of } \\
\text { Total Stops }\end{array}$ \\
\hline$<10$ & 89 & $76 \%$ & $<1$ & $67 \%$ \\
10 to 20 & 3 & $3 \%$ & 1 to 4 & $16 \%$ \\
20 to 40 & 1 & $1 \%$ & 4 to 8 & $3 \%$ \\
$>40$ & 23 & $20 \%$ & $>8$ & $14 \%$ \\
\hline
\end{tabular}
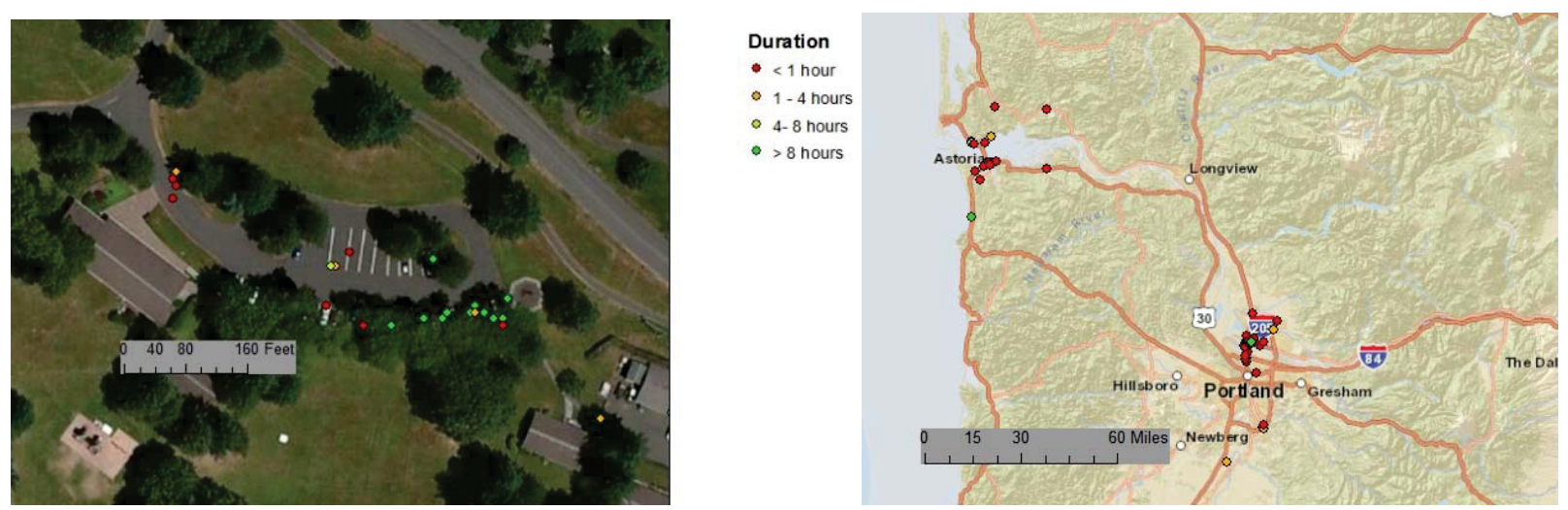

Figure 14. Vehicle G41-4545F all vehicle stops and duration.

Stop locations were typically near the Visitor Center (as expected) and in the vicinity of Astoria, Oregon.

\subsubsection{Pool Vehicle Daily Summary}

The average travel distance per day for the pool vehicle was 39.3 miles. Figure 15 shows that on $84 \%$ of vehicle days, the daily travel was less than the 70 miles considered to be within the BEV safe range. That is, while BEV range can vary based on several factors, most BEVs provide at least 70 miles of 
vehicle range on a single battery charge. Sixteen percent of the pool vehicle's daily travel was greater than 70 miles. Figure 16 identifies that over $40 \%$ of pool vehicle daily travel time is less than 50 minutes.

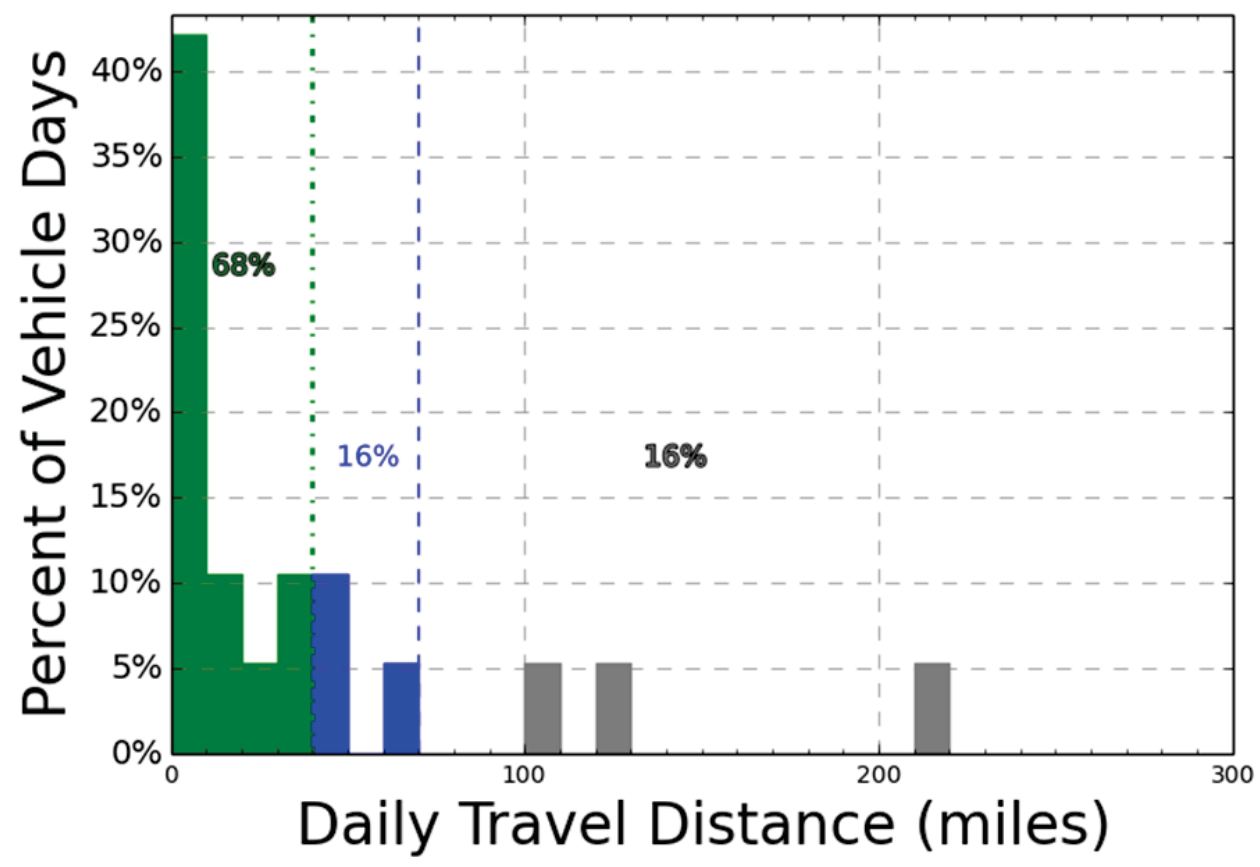

$\ldots$ All days less than 40 miles $\quad--$ All days less than 70 miles

Figure 15. Pool vehicle daily travel miles.

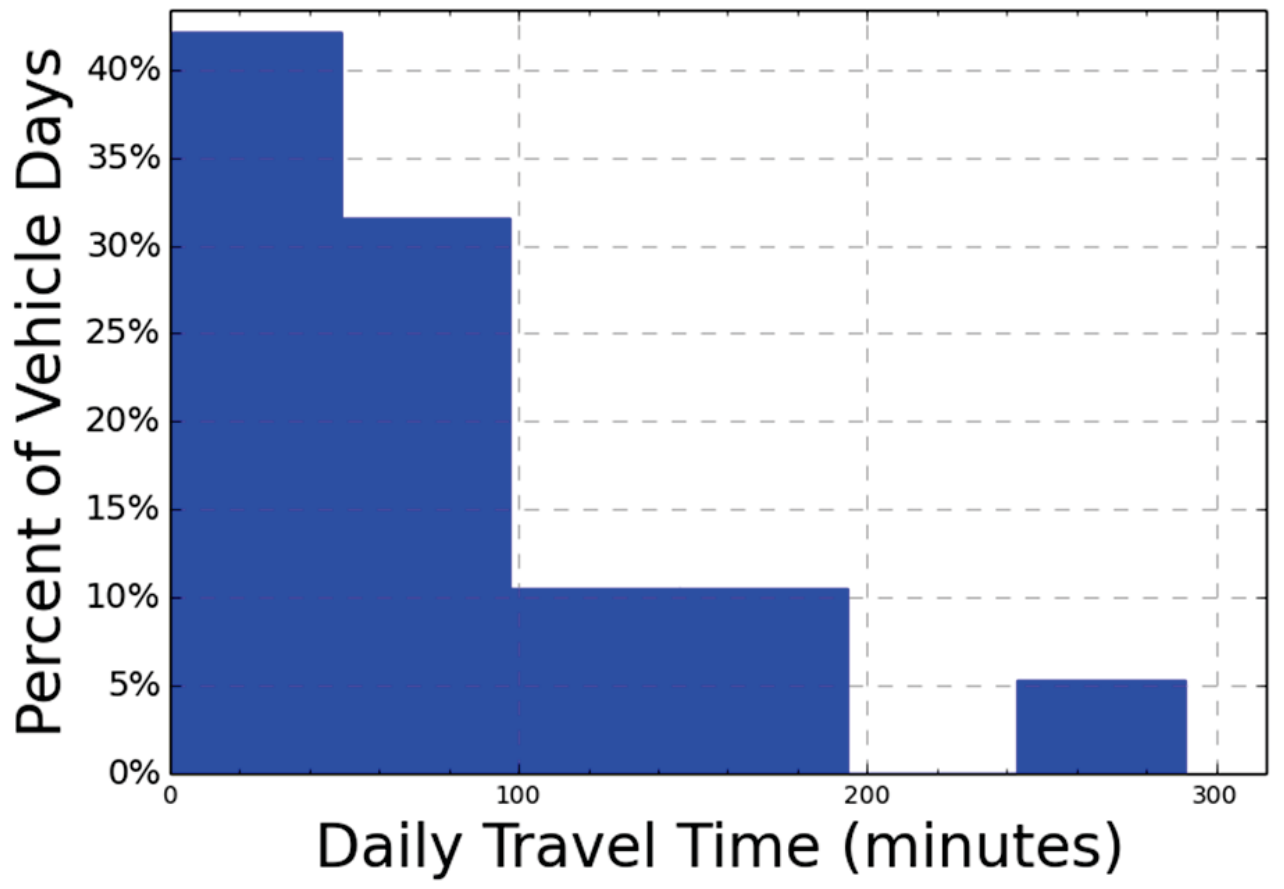

Figure 16. Pool vehicle daily travel time.

Daily travel distances and times are important for identifying the maximum amount of usage during a single day if additional charge opportunities are not available. However, a single day's travel could consist of several short trips and outings. Therefore, reviewing the outing information also is important. If 
charge opportunities are unavailable other than at the home base, a BEV must be able to complete the outing on a single charge. Figure 17 provides the summary of outings for the pool vehicle.

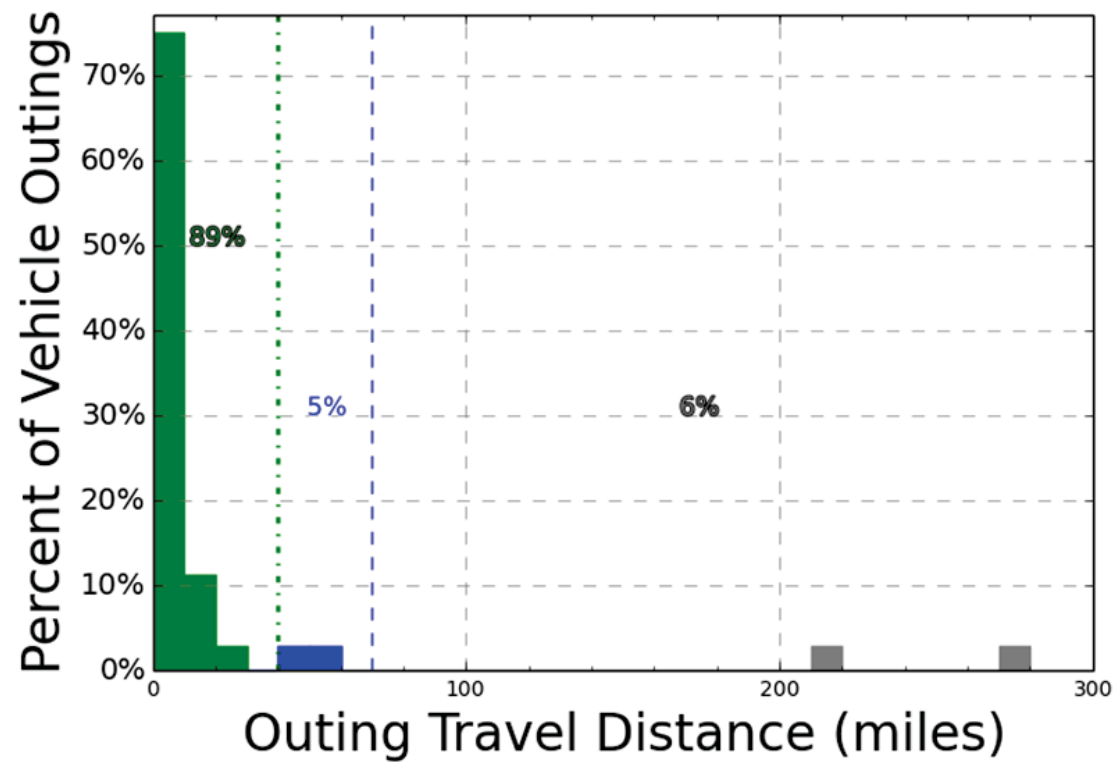

-.. All outings less than 40 miles $\quad--$ All outings less than 70 miles

Figure 17. Pool vehicle outing distance.

\subsubsection{Pool Vehicle Observations/Summary}

The longest single day of travel was 213.7 miles, occurring on February 23, 2013. This day involved a trip to locations in Oregon exceeding 80 miles on a single trip. The longest single outing of 278 miles occurred from March 1 through March 3, with the outing spanning several days and involving a trip to a location in Oregon that was farther than 70 miles from the home base.

This vehicle exceeds the typical BEV safe range limitation on $16 \%$ of vehicle travel days. These data indicate that choosing a BEV for this duty does not seem practical because trips can exceed the published range of most BEVs. A PHEV could meet the pool mission of this vehicle. While composition of the pool fleet is unknown, a combination of BEVs and PHEVs may be desirable for this fleet if vehicle assignments are made knowing the final destination for the vehicle.

There appear to be three choices for FVNHS in implementing PEVs into the pool vehicle fleet. ITSNA notes that the optimum goal would be to incorporate as many BEVs as possible to maximize the advantages of reduced petroleum usage and reduced emissions of GHG.

1. All BEV fleet: While some BEV manufacturers report vehicle range exceeding 70 miles, ITSNA recommends careful evaluation of experienced range to ensure vehicle missions are accomplished. Nevertheless, assuming the 70-mile safe range for a BEV, an all BEV fleet is possible if intermediate charging stations at frequent stop locations provide additional charge opportunities for the pool vehicles assigned to long travel outings. ITSNA notes that the Portland/Vancouver area has a significant density of publicly available EVSE.

2. Mixed BEV/PHEV fleet: Certainly, PHEVs can accomplish the same mission as the current fleet when only considering travel times and distances because the PHEV's gasoline engine can provide motive power after the battery depletes. The data revealed that on $89 \%$ of all vehicle outings, the total travel is less than 40 miles, which typically is the maximum distance a PHEV will travel on battery power only. This scenario requires fleet manager or driver attention to select vehicles appropriately for the anticipated use on that day. 
3. All PHEV fleet: As noted above, PHEVs can accomplish the same mission as the current fleet when only considering travel times and distances. Replacing all current vehicles with PHEVs only requires an evaluation of the individual vehicle's capabilities of currently available PHEVs to meet current pool requirements. Data show that for a significant number of days, the PHEV will operate in pure electric mode. Intermediate charging opportunities provide increased usage of the pure electric mode. Data show significant charging opportunities throughout the day during stop times.

While it would appear that PEVs are suitable replacements for this pool vehicle, additional mission analysis and management input is required. The mission of this and other pool vehicles likely includes considerations other than mileage (such as power demands placed on the vehicle).

The vehicle summary shows sufficient time for charging at the base location during the course of the day and additional opportunities at intermediate charging stations. These stations also provide charging opportunities for the visiting public, whose fees may assist in offsetting operating costs.

ITSNA suggests further mission evaluation be given to pool vehicles when considering the adoption of BEVs and PHEVs. Section 4.4 provides information on PEV sport utility vehicles and sedans currently or soon to be available in the automotive market. However, based on this travel information and providing a conservative approach to the pool fleet, ITSNA suggests that PHEVs meet the current mission requirements. Additional BEVs may be possible with further management or fleet software attention.

Further, with approximately $89 \%$ of the travel outings being less than 40 miles, up to $89 \%$ of the PHEV travel could be electric drive only provided that recharging of the PHEV occurs between the outings. Using intermediate charging along the outing route adds to electric drive only mode.

\subsubsection{Pool Vehicle Charging Needs}

At the time of this writing, no available PHEVs provide for DC fast charging nor do the data suggest that this would be a significant benefit for PHEVs in the pool fleet.

As noted in Section 4, AC Level 2 overnight charging of BEVs is typical, whereas overnight charging of PHEVs uses AC Level 1 outlets. Opportunity charging at intermediate stops obtains the greater benefits from AC Level 2 EVSE. Table 13 identifies the pool vehicle's stop locations of durations greater than 2 hours other than at FVNHS.

Table 13. Location of pool vehicle's frequent stops.

\begin{tabular}{lcc}
\hline Item & Location & Quantity of Stops \\
\hline 1 & Gearhart, OR & 2 \\
2 & Naselle, WA & 2 \\
3 & Hubbard, OR & 1 \\
4 & Oregon City, OR & 1 \\
5 & Astoria, OR & 1 \\
\hline
\end{tabular}

FVNHS identified the Visitor Center as the home base for the pool vehicle. Figure 14 shows the locations of the five locations identified in Table 13.

ITSNA's experience suggests that each vehicle have an assigned overnight charging location at their home base. Assigned stations require less management attention to ensure the completion of overnight charging. BEVs and PHEVs not assigned to these locations also benefit from opportunity charging during visits to the location as part of their normal operation. For this pool vehicle, ITSNA recommends an AC Level 1 outlet for its home base if a PHEV replaces the ICE vehicle.

At times, fleet vehicles obtain benefits from using public charging infrastructure. Figure 18 displays the availability of public charging in the FVNHS area at the time of this writing. The green-colored sites are AC sites, while orange-colored sites are DC fast charge sites. This map provides both AC Level 1 and Level 2 public locations. Upon inclusion of PEVs into the fleet, websites (such as that identified in 
Figure 18) are helpful in determining AC Level 1 or 2, and whether EVSE is available, in use, or out of service. The greater Portland area is an area of highly concentrated, publicly accessible EVSE.

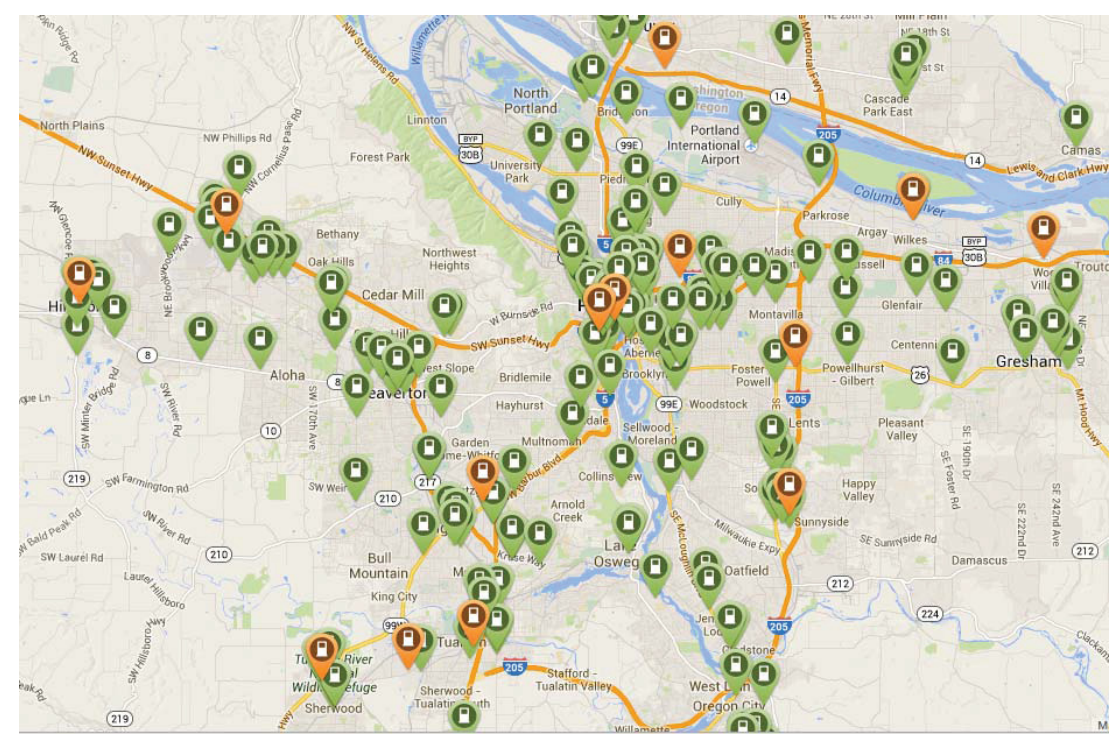

Figure 18. Public charging electric vehicle supply equipment in the Fort Vancouver National Historic Site area. $^{23}$

\subsection{Support Vehicles Evaluation}

Support vehicles provide a specific work function that facilitates the mission of a particular group. The vehicles generally are passenger or light-duty pickup trucks and may include after-market modifications to support the mission. While assigned to maintenance and service areas, missions may vary depending on agency needs. FVNHS identified two support vehicles that supported maintenance activities.

\subsubsection{Summary for Support Vehicles}

This section details the data available for the support vehicle (see Tables 14 and 15).

Table 14. Support vehicle details.

\begin{tabular}{|c|c|c|}
\hline 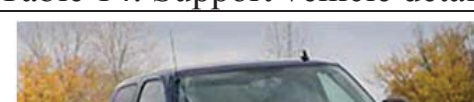 & Make/Model/Year & $\begin{array}{c}\text { Chevrolet/1500 Silverado } \\
\text { Hybrid/2007 }\end{array}$ \\
\hline$z^{z}$ & EPA Class Size & Standard Pickup Truck \\
\hline$=$ & Mission & Support \\
\hline $2=$ & Parking Location & Maintenance Shop \\
\hline$(6)$ & Fleet Vehicle ID & G42-1954F \\
\hline & Fuel Type & Gas \\
\hline www.edmunds.com & EPA Label/MPG (City/Hwy/Combined) & $16 / 19 / 17$ \\
\hline & EPA GHG Emissions (Grams $\left.\mathrm{CO}_{2} / \mathrm{Mi}\right)$ & 523 \\
\hline & Study ID & Logger 79 \\
\hline & Total Vehicle Days/Total Study Days & $47 / 47$ \\
\hline
\end{tabular}

Logger 79 collected data on this vehicle for a period of 47 study days, all of which showed usage. Data validation occurred on $99.5 \%$ of the vehicle data, while $0.5 \%$ of the data are null values. The vehicle's primary home base is located at the Maintenance Center near 401 E. $5^{\text {th }}$ Street.

${ }^{23}$ http://www.plugshare.com/ [accessed February 1, 2014] 
Table 15. Support vehicle travel summary.

\begin{tabular}{lcccc}
\hline \multicolumn{4}{c}{ Vehicle G42-1954F Travel Summary } & \\
& $\begin{array}{c}\text { Per Day } \\
\text { Average/Peak }\end{array}$ & $\begin{array}{c}\text { Per Outing } \\
\text { Average/Peak }\end{array}$ & $\begin{array}{c}\text { Per Trip } \\
\text { Average/Peak }\end{array}$ & Total \\
\hline Travel Distance (Miles) & $19.3 / 67.2$ & $1.8 / 49.9$ & $5.9 / 22.5$ & 908 \\
Travel Time (Minutes) & $73.6 / 143$ & $38.9 / 67$ & $11.2 / 26$ & 3,460 \\
Idle Time (Minutes) & $4.7 / \mathrm{NA}$ & $2.5 / \mathrm{NA}$ & $.7 / \mathrm{NA}$ & 348 \\
\hline
\end{tabular}

During the study period, the vehicle traveled a total distance of 908 miles and logged 58 hours of driving usage. Figures 19 and 20 show the travel summary for the support vehicle by daily mileage and daily usage time.

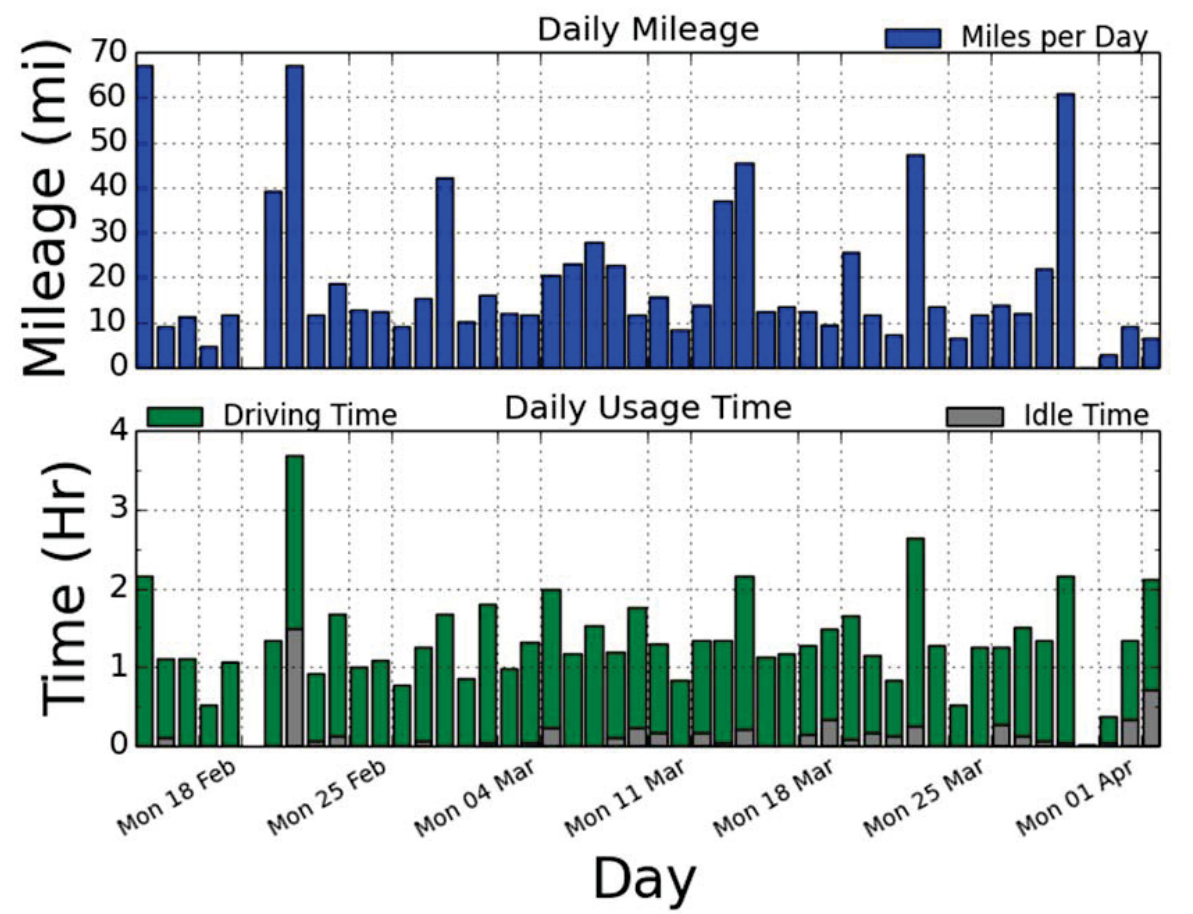

Figure 19. Support vehicle daily mileage and usage time.

Figure 20 identifies the hours of the day that the vehicle typically was in use.

Stop information identified where the vehicle parked for a significant amount of time. EVSE, if placed at highly utilized stop locations, provides additional charging opportunities for PEVs. Figure 21 shows the primary vehicle stop locations both locally and in the general area. Table 16 shows the stop distances and duration for the support vehicle. Stop locations typically are near the maintenance area (as expected) and in the vicinity of Portland, Oregon. 


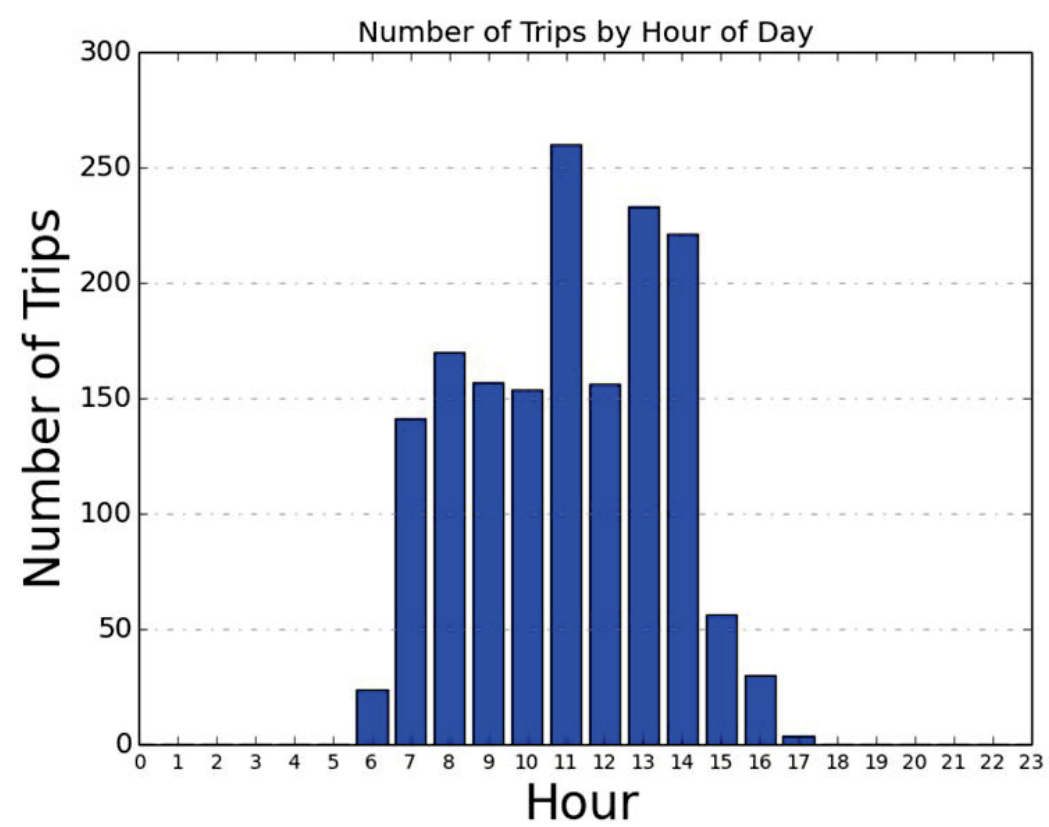

Figure 20. Support vehicle daily usage time.

Table 16. Support vehicle stop information.

\begin{tabular}{ccccc}
\hline \multicolumn{2}{c}{ Stop Distance from Home Base } & \multicolumn{2}{c}{ Stop Duration } \\
$\begin{array}{c}\text { Distance From } \\
\text { Home Base (Miles) }\end{array}$ & Stops & Percentage of Total & Stop Duration (Hours) & $\begin{array}{c}\text { Percentage of } \\
\text { Total Stops }\end{array}$ \\
\hline$<10$ & 704 & $98.5 \%$ & $<1$ & $84 \%$ \\
10 to 20 & 11 & $1.5 \%$ & 1 to 4 & $10 \%$ \\
20 to 40 & 0 & 0 & 4 to 8 & 0 \\
$>40$ & 0 & 0 & $>8$ & $6 \%$ \\
\hline
\end{tabular}
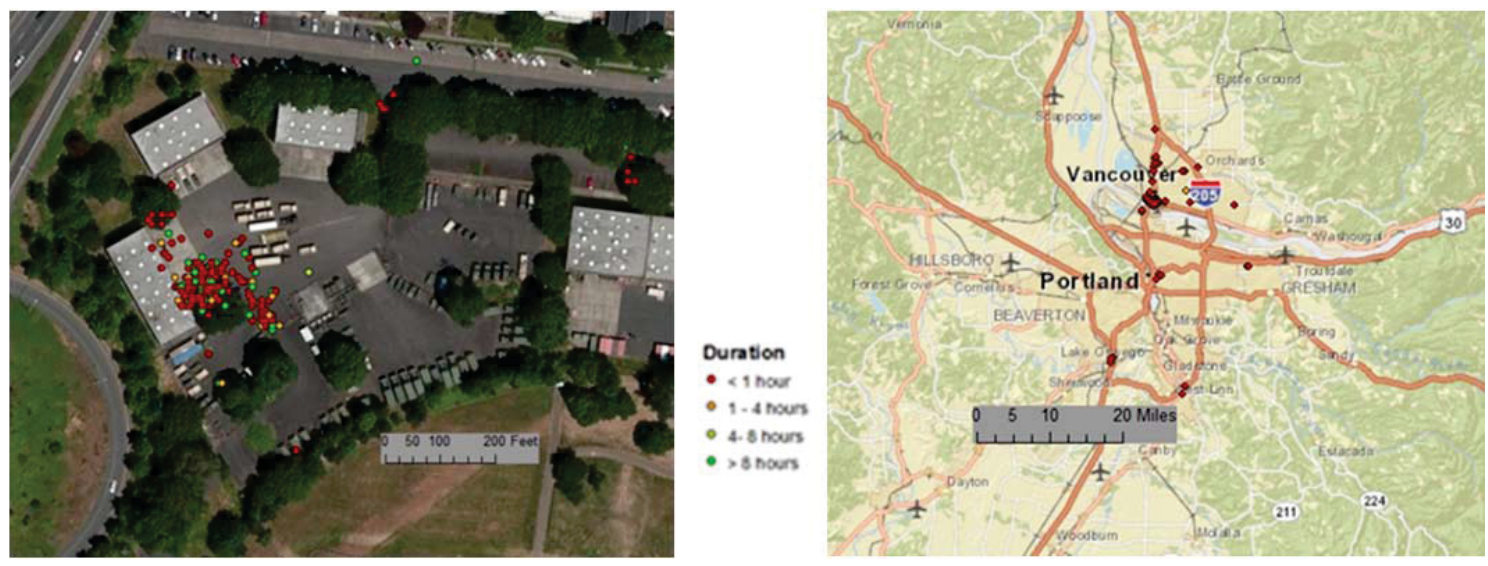

Figure 21. Vehicle G41-4545F all vehicle stops.

\subsubsection{Support Vehicle Daily Summary}

The average travel distance per day for the support vehicle was 19.3 miles. Figure 22 shows that on all vehicle days, the daily travel was less than the 70 miles, which is considered to be within the BEV safe range. That is, while BEV range can vary based on several factors, most BEVs provide at least 70 miles 
of vehicle range on a single battery charge. While Figure 19 shows the vehicle operated nearly every day, that figure and Figure 23 show that the total daily usage time was less than 3 hours per day.

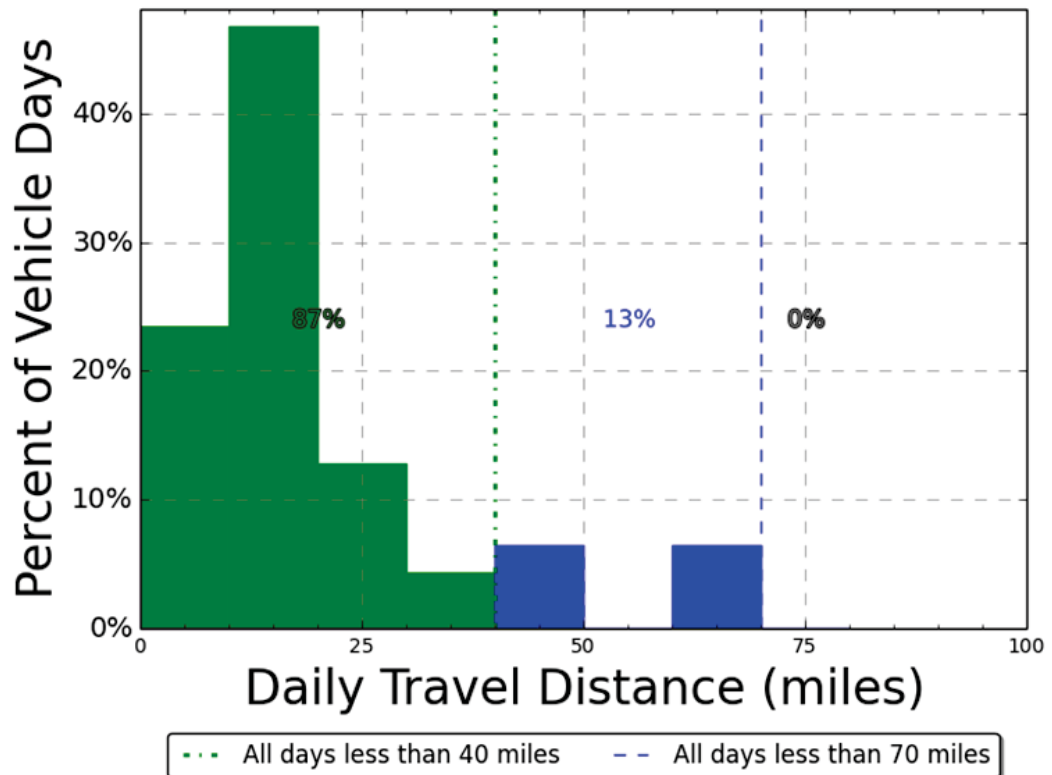

Figure 22. Support vehicle daily travel miles.

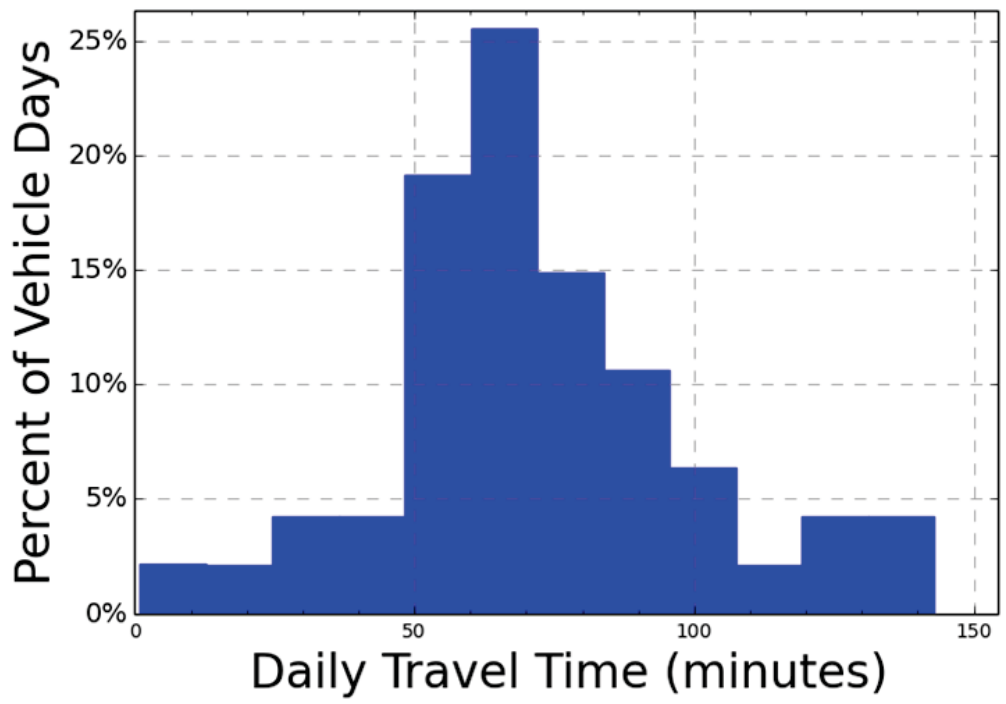

Figure 23. Support vehicle daily travel time.

Daily travel distances and times are important to identify the maximum amount of usage during a single day if additional charge opportunities are not available. However, a single day's travel could consist of several short trips and outings. Thus, reviewing the outing information is also important. If charge opportunities are unavailable other than at the home base, a BEV must be able to complete the outing on a single charge. Figure 24 provides the summary of outings for the support vehicle.

The longest single day travel was 67 miles and occurred on February 13, 2013. It also was the day with the longest single outing of 49.9 miles, involving a trip to a location in Oregon City about 18 miles from the home base. (Note that this one outing accounts for less than $0.5 \%$ of all outings.) That day also included several short trips close to the home base. 


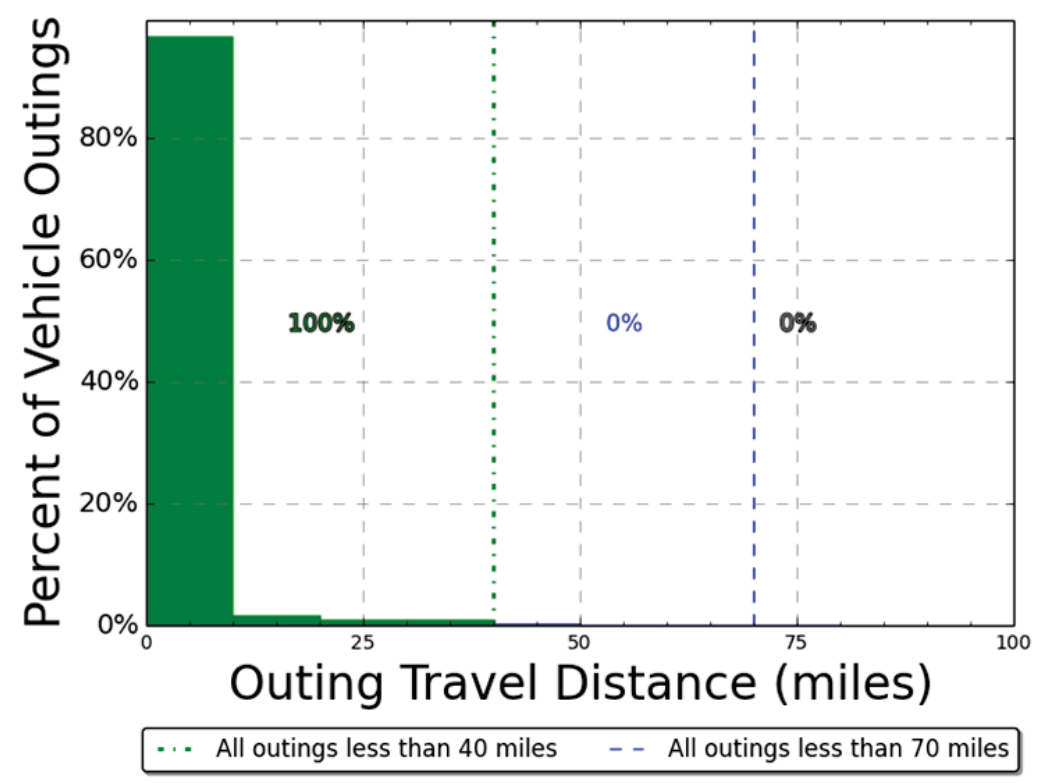

Figure 24. Support vehicle outing distance.

\subsubsection{Support Vehicle Observations/Summary}

This support vehicle had no days when daily travel distances exceeded 70 miles. One outing experience exceeded 40 miles.

As before, there appear to be three choices for FVNHS in implementing PEVs into the support vehicle fleet. ITSNA notes that the optimum goal would be to incorporate as many BEVs as possible to maximize the advantages of reduced petroleum usage and reduced emissions of GHG.

1. All BEV fleet: While some BEV manufacturers report a vehicle range exceeding 70 miles, ITSNA recommends careful evaluation of experienced range to ensure vehicle missions are accomplished. Nevertheless, assuming the 70-mile safe range for a BEV, a BEV is a suitable replacement for this support vehicle without additional considerations such as intermediate charging locations.

2. Mixed BEV/PHEV fleet: Certainly, PHEVs can accomplish the same mission as the current fleet when only considering travel times and distances, because the PEV's gasoline engine can provide motive power when the battery depletes. The data reveal that for all but one of vehicle travel days, the total travel is less than the 40 miles, which typically is the maximum distance a PHEV will travel on battery power only. Intermediate charging locations provide the recharge necessary to increase the all-electric drive. Sufficient daily stop time exists to recharge if EVSE are convenient.

3. All PHEV fleet: As noted above, PHEV can accomplish the same mission as the current fleet when only considering travel times and distances. Replacing all current vehicles with PHEV only requires an evaluation of the individual vehicle's capabilities of currently available PHEVs to meet current support vehicle requirements.

While it would appear that a BEVs is a suitable replacement for this support vehicle, additional mission analysis and management input is required. The mission of these vehicles likely includes considerations other than mileage, such as power demands and the load-carrying capabilities placed on the vehicle.

The vehicle summary shows sufficient time for charging at the base location during the course of the day and additional opportunities at intermediate charging stations. These stations also provide charging opportunities for the visiting public, whose fees may assist in offsetting operating costs. 
ITSNA suggests further mission evaluation be given to support vehicles when considering the adoption of BEVs and PHEVs. However, based on this travel information and providing a conservative approach to the support fleet, ITSNA suggests that a BEV meets the current mission requirements.

The support vehicle in this study was a pickup truck. Section 4.4 provides information on PEV trucks and vans currently or soon to be available in the automobile market and BEVs that may be considered for replacing this support vehicle. ${ }^{24}$

The total number of support vehicles in this current fleet is unknown. A conservative approach would likely mix some PHEVs into the fleet for an occasional outing that might exceed the capabilities of a $\mathrm{BEV}$. As noted above, some management attention may be required to assign support vehicles based on expected usage.

\subsubsection{Support Vehicle Charging Needs}

The majority of the support vehicle activity occurs during daytime hours, which leaves significant time during the night for recharging. ITSNA suggests replacement of the monitored support vehicle with a BEV. While several BEVs are available with DC fast charging capability, data suggest this would not be a significant benefit for this support fleet vehicle. Additional charging of BEVs during the day is not a requirement, nor would DC fast chargers be required.

As noted in Section 5, AC Level 2 overnight charging of BEVs is typical, whereas overnight charging of PHEVs often utilize AC Level 1 outlets. Opportunity charging at intermediate stops obtains greater benefits from AC Level 2 EVSE.

Analysis of this support vehicle's stop locations greater than a 2-hour duration shows only two stops out of 63 that were not at FVNHS. ITSNA suggests additional charge locations would provide little benefit.

ITSNA's experience suggests that each vehicle have an assigned overnight charging location at the home base. Assigned stations require less management attention to ensure completion of overnight charging. BEVs and PHEVs not assigned to these locations also benefit from opportunity charging during visits to the location as part of normal operation. For this support vehicle, ITSNA recommends an AC Level 2 EVSE for its home base.

\section{GREENHOUSE GAS EMISSIONS AVOIDED AND FUEL COST REDUCTION ANALYSIS}

PEV substitution for an existing conventional vehicle avoids GHG emissions and reduces fuel costs. The GHG emissions avoided occur due to the difference in emissions associated with power plant electricity generation versus fuel combustion that occurs in the engine of a conventional vehicle. This analysis does not account for life-cycle emissions that occur outside of electricity generation and fuel combustion phases (i.e., materials and resource extraction, production supply-chains, and decommissioning are not accounted for). These phases are beyond the scope of this report due to the significant effort required to conduct an accurate environmental life-cycle assessment for a transportation system in a very specific setting. Cost reduction also occurs because the cost of electricity is much less than the cost of gasoline and PEVs are more efficient than conventional ICE vehicles. Because fuel logs were not kept, the mileage accumulated by each vehicle provides the source of fuel consumption estimates for the study vehicles.

In order to perform the analysis, EPA fuel economy ratings are used, ${ }^{25}$ with Tables 17 and 18 providing these ratings. The ratings for the PHEVs in Table 19 include electric-only operation. Because these are estimates, assumptions include the following:

\footnotetext{
${ }^{24}$ Note that Via Motors has recently added PEV pickup trucks to PG\&E in the San Francisco Bay Area: http://www.viamotors.com/blog/national-plugin-day-2012-cupertino/ [accessed July 19, 2013]

${ }^{25}$ http://www.fueleconomy.gov/feg/Find.do?action=sbs\&id=33558 [accessed February 2, 2014]
} 
1. PHEVs operate in electric mode only. This is reasonable for most daily operations, as described in Section 5. This assumption results in savings calculations slightly higher than that realized through the expected operation of combined electric and gasoline motive operations.

2. The fuel economy for the electric only mode of the Mitsubishi Outlander PHEV is assumed to be the same as the Toyota RAV4 electric vehicle, because EPA has not yet created ratings for the former vehicle.

3. The Mitsubishi Outlander replaces the pool minivan and the Toyota RAV4 electric vehicle replaces the support pickup ${ }^{26}$.

Table 17 provides a pictorial view of the replacements involved.

Table 17. Current vehicle replacement plug-in electric vehicles.

$\begin{array}{ll}\text { Pool Vehicle Minivan } & \text { Analysis Replacement PEV } \\ \text { Support Sport Utility } \\ \text { Vehicle/Pickup }\end{array}$

Table 18. Environmental Protection Agency internal combustion engine fuel economy ratings.

\begin{tabular}{|c|c|c|c|c|}
\hline $\begin{array}{l}\frac{0}{0} \\
\frac{0}{0} \\
>\end{array}$ & Mission & Make and Model & $\begin{array}{c}\text { Model } \\
\text { Year }\end{array}$ & $\begin{array}{l}\text { Fuel Economy- } \\
\text { Combined } \\
\text { (miles/gallon) }\end{array}$ \\
\hline 84 & Pool & Chevrolet Uplander & 2007 & 19 \\
\hline 91 & Support & Chevrolet 1500 Silverado & 2007 & 17 \\
\hline
\end{tabular}

Table 19. Environmental Protection Agency plug-in electric vehicle fuel economy ratings.

\begin{tabular}{|c|c|c|c|c|}
\hline Mission & Make and Model & $\begin{array}{l}\text { Model } \\
\text { Year }\end{array}$ & $\begin{array}{l}\text { Fuel Consumption- } \\
\text { Combined (Wh/mile) }\end{array}$ & $\begin{array}{l}\text { Fuel Economy } \\
\text { (MPGe) }\end{array}$ \\
\hline Pool & Mitsubishi Outlander & 2014 & $\begin{array}{c}440 \text { (assumed equal to } \\
\text { Toyota RAV4) }\end{array}$ & 76 \\
\hline Support & Toyota RAV4 Electric Vehicle & 2014 & 440 & 76 \\
\hline
\end{tabular}

${ }^{26}$ Although a sport utility vehicle replaces the support vehicle pickup due to current market availability, PEV pickup trucks will be increasingly available in the near future. One example is the Via Motors VTRUX (http://www.viamotors.com/wpcontent/uploads/VIA-Small-Brochure.pdf). Note that Via Motors has recently provided fleet pickup trucks to PG\&E in the San Francisco Bay Area (http://www.viamotors.com/blog/national-plugin-day-2012-cupertino/) [accessed July 19, 2013]. 
Calculations provided for GHG emissions and fuel savings include both a total U.S. perspective and for the local area. The electricity generation mix of power plants for the total United States is different from the local mix of generation in the Vancouver, Washington area. Likewise, the national average cost for petroleum fuel is different from the local cost for fuel. This analysis includes both approaches in order to allow for local evaluation and to provide the potential benefit for fleet vehicles in other locations of the United States that may be of interest. The final report from ITSNA to Idaho National Laboratory primarily will consider the national figures.

For the GHG emissions avoided portion of the analysis, the GHG emissions (in pounds of carbon dioxide equivalent, which accounts for other GHGs such as methane and nitrous oxide, $l b-\mathrm{CO}_{2} \mathrm{e}$ ) from combustion of gasoline was $20.1 \mathrm{lb}-\mathrm{CO}_{2} \mathrm{e} /$ gallon. ${ }^{27}$ EPA reports $\mathrm{GHG}$ emissions from the production of electricity. The annual report is available in the Emissions and Generation Resource Integrated Database. The most recent publication is for 2010. The electric utility in the Vancouver area is Clark Public Utilities. The generation mix was reported in the 2012 Integrated Resource Plan. ${ }^{28}$ Using this mix and the Emissions and Generation Resource Integrated Database plant reports, Clark Public Utilities emissions for 2010 for the production of electricity were $0.58 \mathrm{lb}-\mathrm{CO}_{2} / \mathrm{kWh}$.

The U.S. average for GHG emissions for the production of electricity are $1.53 \mathrm{lb}-\mathrm{CO}_{2} \mathrm{e} / \mathrm{kWh}^{29}$. Therefore, GHG emissions avoided are the annual GHG emitted by the current vehicle (total annual gallons gasoline $\times$ GHG emissions/gallon) minus the annual GHG emitted by the replacement PEV (total annual $\mathrm{kWh} \times \mathrm{GHG}$ emissions $/ \mathrm{kWh}$ ).

For the cost-avoided piece of the analysis, national fuel cost assumptions are $\$ 3.46 /$ gallon for gasoline with the Oregon/Washington Area very close to the national average ${ }^{30}$. Electrical costs assumptions are $0.13 \$ / \mathrm{kWh}$ for the United States and $0.05 \$ / \mathrm{kWh}$ for the Vancouver area ${ }^{31}$. Fuel costs savings are the current vehicle calculated annual gasoline cost (total annual gallons gasoline $\times$ cost/gallon) minus the electricity cost (total annual $\mathrm{kWh} \times \operatorname{cost} / \mathrm{kWh}$ ) of the replacement PEV traveling the same distance.

Extrapolation of the actual total distance traveled by each vehicle during the study days to 365 days yields estimates for yearly GHG emissions avoided and fuel cost reductions. The results of this analysis (shown in Table 20) demonstrate that the substitution of a conventional ICE vehicle with a PEV can reduce GHG emissions and fuel costs dramatically. The benefits in the Vancouver area are greater than the national averages because of the lower local GHG production and the lower cost of electrical energy.

Table 20. Greenhouse gas emissions avoidance and fuel cost reduction analysis summary.

\begin{tabular}{|c|c|c|c|c|c|c|}
\hline Vehicle & Mission & $\begin{array}{c}\text { Make and } \\
\text { Model }\end{array}$ & $\begin{array}{c}\text { Extrapolated } \\
\text { United States } \\
\text { Yearly } \mathrm{CO}_{2} \mathrm{e} \\
\text { Avoided (lb- } \\
\mathrm{CO}_{2} \mathrm{e} / \text { year) }\end{array}$ & $\begin{array}{c}\text { Extrapolated } \\
\text { Local_Yearly } \\
\mathrm{CO}_{2} \mathrm{e} \text { Avoided } \\
\text { (lb- } \mathrm{CO}_{2} \mathrm{e} / \text { year) }\end{array}$ & $\begin{array}{l}\text { Extrapolated } \\
\text { United States } \\
\text { Yearly Fuel Cost } \\
\text { Reduction } \\
\text { (\$/year) }\end{array}$ & $\begin{array}{l}\text { Extrapolated Local } \\
\text { Yearly Fuel Cost } \\
\text { Reduction (\$/year) }\end{array}$ \\
\hline G41-4545F & Pool & $\begin{array}{l}\text { Chevrolet, } \\
\text { Uplander }\end{array}$ & $2,278(26 \%)$ & $4,754(76 \%)$ & $\$ 739.75$ & $\$ 948.23$ \\
\hline G42-1954F & Support & $\begin{array}{l}\text { Chevrolet, } \\
1500 \\
\text { Silverado }\end{array}$ & $3,591(43 \%)$ & $6,540(78 \%)$ & $\$ 1,032.18$ & $\$ 1,280.48$ \\
\hline
\end{tabular}

\footnotetext{
${ }^{27} \mathrm{http}$ //avt.inel.gov/pdf/EVProj/106077-891082.ghg.pdf for the methodology for gasoline [accessed April 10, 2013].

${ }^{28} \mathrm{http}$ ://www.clarkpublicutilities.com/index.cfm/aboutus/ourpowersupply/archives/2012-irp/ [accessed March 5, 2014]

${ }^{29}$ http://avt.inel.gov/pdf/EVProj/106077-891082.ghg.pdf [accessed April 10, 2013]

${ }^{30} \mathrm{http}$ //fuelgaugereport.aaa.com/?redirectto=http://fuelgaugereport.opisnet.com/index.asp [accessed March 4, 2014]

${ }^{31}$ http://www.clarkpublicutilities.com/index.cfm/your-business/rates-and-billing/industrial-electric-rates/ [accessed March 5, 2014]
} 


\section{OBSERVATIONS}

ITSNA appreciates the opportunity to present the results of this evaluation. Observations for possible follow-up action include the following:

\section{Observation \#1:}

Implementation: FVNHS may move forward in the near future with replacement of the pool and support vehicles with PEVs as current budget and vehicle replacement schedules allow. Certainly, the vehicle types studied in this report are candidates for immediate replacement.

\section{Observation \#2:}

Fleet Inventory: A more thorough examination of the quantities and types of fleet vehicles within each usage category may be beneficial to quantify the potential for replacement by PEVs. While ITSNA suggests a mix of BEVs and PHEVs, a more refined look may be possible. In addition, this study did not look at the other fleet vehicle categories such as enforcement, specialty, transport vehicles, and shuttles/buses.

\section{Observation \#3:}

Vehicle Replacement Plan: Development of a detailed vehicle replacement plan could be beneficial. This plan would include the cost and schedule for vehicle replacement. A more detailed survey and calculation of the use of the fleet vehicles (such as vehicle parking locations, age of vehicle, expected replacement time, expected replacement costs, GSA vehicle costs, EVSE cost, and EVSE installation costs) would provide support to this replacement plan. The savings in GHG emissions, petroleum usage reduction, and fuel cost savings flow from this detailed plan.

\section{Observation \#4:}

Infrastructure Planning: In conjunction with the replacement plan, evaluation of the FVNHS site for placement of PEV charge infrastructure could be beneficial. ITSNA has significant experience in this area and plans would consider not only fleet vehicle charging needs, but also the convenience and benefits that charge infrastructure would provide for employees or visitors. This planning also considers the existing facility electrical distribution system. Vehicle home base considerations factor into the ratio of PEVs to EVSE units to maintain all vehicles at operational readiness.

Charging stations located at various destination points provide adequate infrastructure for PEV charging of the FVNHS fleet. Opportunity also exists to support public use of PEVs. Portland and Vancouver drivers are leaders in the adoption of PEVs. ${ }^{32}$ Charging stations at FVNHS provide for opportunity charging by the public. FVNHS benefits through the collection of charging fees during times when these stations are not required for the overnight charging of fleet vehicles. The fees avoid questions of the federal agency providing fuel for privately owned vehicles and yet support the costs for installation and operation of the EVSE.

\footnotetext{
${ }^{32}$ http://avt.inel.gov/evproject.shtml\# [accessed April 11, 2013].
} 


\section{Appendix A \\ Fleet Survey Form}

\begin{tabular}{ll} 
& \multicolumn{1}{c}{ Fleet Survey Sample } \\
\hline Project Name: & BEA-FEMP Fleet II \\
Agency Name: & National Park Service \\
Location: & Fort Vancouver National Historical Site (FVNHS) \\
Date Requested: & \\
\hline
\end{tabular}

The following survey questions are used to lead the discussion concerning the mission of the current fleet of vehicles. If responding by e-mail, please use one form for each vehicle.

Please submit the data sheets to Ian Nienhueser at ian.nienhueser@intertek.com by fax at (602) 443-9007. If you have questions, please contact Ian at the email above or by phone at (702) 738-2706.

\begin{tabular}{ll}
\hline & Vehicle Information \\
\hline Today's Date: & Odometer Reading: \\
Make: & Data Logger ID: \\
Model: & Data Logger Installed: \\
Year: & Fuel Type: \\
VIN: & Miles per Gallon: \\
Agency Fleet ID: & Miles per Year: \\
\hline
\end{tabular}

1. Vehicle Mission:

$\begin{array}{ll}\square & \text { Pool Vehicle } \\ \square & \text { Enforcement Vehicle } \\ \square & \text { Support Vehicle } \\ \square & \text { Transport Vehicle } \\ \square & \text { Specialty Vehicle } \\ \square & \text { Shuttle/Bus } \\ \square & \text { Low Speed Vehicle }\end{array}$

2. Vehicle Typical Parking Location: (parking lot name/designation, nearest building number) 


\section{Appendix B Vehicle Characterization}

Table B-1. Fort Vancouver National Historic Site vehicle index.

\begin{tabular}{cccccc}
\hline \multicolumn{1}{c}{ ID } & Make & Model & Year & Data Logger & Mission \\
\hline G41-4545F & Chevrolet & Uplander & 2007 & 77 & Pool \\
G42-1954F & Chevrolet & 1500 Silverado Hybrid & 2007 & 79 & Support \\
G63-2424L* & Dodge & Ram 2500 & 2011 & 80 & Support \\
\hline
\end{tabular}

*vehicle provided no data 


\section{Appendix C Definitions}

Alternative fuel

City fuel economy (MPG)

Conventional fuel

Daily travel

Diesel fuel

E85

Electric vehicle

Ethanol-fueled vehicle

Federal vehicle standards

Government motor vehicle

Gross vehicle weight

rating

GSA fleet
An alternative fuel means any fuel other than gasoline and diesel fuels, such as methanol, ethanol, and gaseous fuels (40 CFR 86.1803-01). A fuel type other than petroleum-based gasoline or diesel as defined by the Energy Policy Act (examples include ethanol, methanol, compressed natural gas, propane, and electrical energy).

City fuel economy means the city fuel economy determined by operating a vehicle (or vehicles) over the driving schedule in the federal emission test procedure or determined according to the vehicle-specific 5-cycle or derived 5-cycle procedures (40 CFR 600.001).

A petroleum-based fuel (examples include gasoline and diesel fuel). The sum of daily trips and stops in one day.

Diesel means a type of engine with operating characteristics significantly similar to the theoretical diesel combustion cycle. The non-use of a throttle during normal operation is indicative of a diesel engine (49 CFR 86-1803).

Ethanol fuel blend of up to $85 \%$ denatured ethanol fuel and gasoline or other hydrocarbons by volume.

Electric vehicle means a motor vehicle that is powered solely by an electric motor drawing current from a rechargeable energy storage system, such as from storage batteries or other portable electrical energy storage devices, including hydrogen fuel cells, provided that

(1) The vehicle is capable of drawing recharge energy from a source off the vehicle, such as residential electric service

(2) The vehicle must be certified to the emission standards of Bin \#1 of Table S04-1 in § 86.1811-09(c)(6)

(3) The vehicle does not have an onboard combustion engine/generator system as a means of providing electrical energy (40 CFR 86-1803).

Ethanol-fueled vehicle-means any motor vehicle or motor vehicle engine that is engineered and designed to be operated using ethanol fuel (i.e., a fuel that contains at least $50 \%$ ethanol $\left(\mathrm{C}_{2} \mathrm{H}_{5} \mathrm{OH}\right)$ by volume) as fuel (40 CFR 86.1803-01).

The document that establishes classifications for various types and sizes of vehicles, general requirements, and equipment options. It is issued annually by the GSA Vehicle Acquisition and Leasing Service's Automotive Division.

Any motor vehicle that the government owns or leases. This includes motor vehicles obtained through purchase, excess, forfeiture, commercial lease, or GSA fleet lease.

Gross vehicle weight rating (GVWR) means the value specified by the vehicle manufacturer as the maximum design loaded weight of a single vehicle (e.g., vocational vehicle) (US Government Printing Office 2009)

GSA fleet lease means obtaining a motor vehicle from the General Services Administration fleet (GSA fleet) (41 CFR 102-34). 
Heavy light-duty truck

Highway fuel economy (Hwy MPG)

Hybrid electric vehicle

Idle time

Law enforcement

Light-duty motor vehicle

Light-duty truck
Heavy light-duty truck means any light-duty truck rated greater than 6,000 lb GVWR. The light-duty truck 3 (LDT3) and LDT4 classifications comprise the heavy light-duty truck category (40 CFR 86.1803-01).

Highway fuel economy means the highway fuel economy determined either by operating a vehicle (or vehicles) over the driving schedule in the federal highway fuel economy test procedure or determined according to either the vehicle-specific, 5-cycle equation, or the derived 5-cycle equation for highway fuel economy (40 CFR 600.001).

Hybrid electric vehicle means a motor vehicle that draws propulsion energy from onboard sources of stored energy that are both an internal combustion engine or heat engine using consumable fuel and a rechargeable energy storage system (such as a battery, capacitor, hydraulic accumulator, or flywheel), where recharge energy for the energy storage system comes solely from sources on board the vehicle.

Idle time is logged whenever a vehicle idles with the engine running for 3 minutes or longer.

Law enforcement motor vehicle means a light-duty motor vehicle that is specifically approved in an agency-s appropriation act for use in apprehension, surveillance, police, or other law enforcement work or specifically designed for use in law enforcement. If not identified in an agency's appropriation language, a motor vehicle qualifies as a law enforcement motor vehicle only in the following cases:

(1) A passenger automobile having heavy-duty components for electrical, cooling, and suspension systems and at least the next higher cubic inch displacement or more powerful engine than is standard for the automobile concerned

(2) A light truck having emergency warning lights and identified with markings such as "police"

(3) An unmarked motor vehicle certified by the agency head as essential for the safe and efficient performance of intelligence, counterintelligence, protective, or other law enforcement duties

(4) A forfeited motor vehicle seized by a federal agency that subsequently is used for performing law enforcement activities (41 CFR Part 10234.35).

Any motor vehicle with a GVWR of 8,500 pounds or less (41 CFR 102$34)$.

Light-duty truck means any motor vehicle rated at 8,500 pounds GVWR or less, which has a curb weight of 6,000 pounds or less and, which has a basic vehicle frontal area of 45 square feet or less, which is as follows:

(1) Designed primarily for purposes of transportation of property or is a derivation of such a vehicle

(2) Designed primarily for transportation of persons and has a capacity of more than 12 persons

(3) Available with special features, enabling off-street or off-highway operation and use.

LDT1 means any light light-duty truck up through 3,750-lb loaded vehicle weight.

LDT2 means any light light-duty truck greater than 3,750-lb loaded vehicle weight.

LDT3 means any heavy light-duty truck up through 5,750-lb adjusted 
Light-duty vehicle

Low-speed vehicle

Medium-duty passenger vehicle

Model year

$M P G$

MPGe

Non-passenger automobile loaded vehicle weight.

LDT4 means any heavy light-duty truck greater than 5,750-1b adjusted loaded vehicle weight (US Government Printing Office 2009)

Light-duty vehicle means a passenger car or passenger car derivative capable of seating 12 passengers or less.

Low-speed vehicle means a motor vehicle

(1) That is 4-wheeled

(2) Whose speed attainable in $1.6 \mathrm{~km}$ ( 1 mile) is more than 32 kilometers per hour (20 miles per hour) and not more than 40 kilometers per hour (25 miles per hour) on a paved level surface

(3) Whose GVWR is less than 1,361 kilograms (3,000 pounds) (49 CFR 571.3 - Definitions).

Medium-duty passenger vehicle means any heavy-duty vehicle (as defined in this subpart) with a GVWR of less than 10,000 pounds that is designed primarily for transportation of persons. The medium-duty passenger vehicle definition does not include any vehicle which

(1) Is an "incomplete truck" as defined in this subpart

(2) Has a seating capacity of more than 12 persons

(3) Is designed for more than 9 persons in seating rearward of the driver's seat

(4) Is equipped with an open cargo area (for example, a pick-up truck box or bed) of 72.0 inches in interior length or more. A covered box not readily accessible from the passenger compartment will be considered an open cargo area for purposes of this definition (US Government Printing Office 2009)

Model year means the manufacturer's annual production period (as determined by the administrator), which includes January 1 of such calendar year; provided that if the manufacturer has no annual production period, the term "model year" shall mean the calendar year (40 CFR 86-1803.01).

"MPG" or "mpg" means miles per gallon. This generally may be used to describe fuel economy as a quantity or it may be used as the units associated with a particular value.

MPGe means miles per gallon equivalent. This generally is used to quantify a fuel economy value for vehicles that use a fuel other than gasoline. The value represents miles the vehicle can drive with the energy equivalent of one gallon of gasoline:

(c) SCF means standard cubic feet

(d) SUV means sport utility vehicle

(e) CREE means carbon-related exhaust emissions [76 FR 39527, July 6, 2011].

A non-passenger automobile means an automobile that is not a passenger automobile or a work truck and includes vehicles described in paragraphs (a) and (b) of 49 CFR 523.5. 
Owning agency

Passenger automobile

Pickup truck

Plug-in hybrid electric vehicle

Vehicle class

Vehicle configuration

Vehicle days

Vehicle home base

Vehicle study period
Owning agency means the executive agency that holds the vehicle title, manufacturer's Certificate of Origin or is the lessee of a commercial lease. This term does not apply to agencies that lease motor vehicles from the GSA fleet (41 CFR Part 102-34.35).

A passenger automobile is any automobile (other than an automobile capable of off-highway operation) manufactured primarily for use in the transportation of not more than 10 individuals (49 CFR 523.4 - Passenger automobile). A sedan or station wagon designed primarily to transport people (41 CFR 102-34).

Pickup truck means a non-passenger automobile, which has a passenger compartment and an open cargo bed (49 CFR 523.2).

PHEV means a hybrid electric vehicle that has the capability to charge the battery from an off-vehicle electric source, such that the off-vehicle source cannot be connected to the vehicle while the vehicle is in motion (40 CFR 86.1803).

The designation of motor vehicle types that include sedans, station wagons, ambulances, buses, and trucks, or different categories of vehicles according to Federal vehicle standards and further defined in 49 CFR 600.315-82.

Vehicle configuration means a unique combination of basic engine, engine code, inertia weight class, transmission configuration, and axle ratio.

The number of days a vehicle was driven or utilized during the (vehicle) study period.

The primary assigned outing beginning and ending parking location for the vehicle.

The time period the vehicle, within the study, has been equipped with a data logger. 